\title{
Re-design of Precision Signal Conditioning Circuit for detecting Schumann Resonance
}

\author{
Rohith Bikkina \\ rb00003@mix.wvu.edu
}

Follow this and additional works at: https://researchrepository.wvu.edu/etd

Part of the Electrical and Electronics Commons, Signal Processing Commons, and the Systems and Communications Commons

\section{Recommended Citation}

Bikkina, Rohith, "Re-design of Precision Signal Conditioning Circuit for detecting Schumann Resonance" (2021). Graduate Theses, Dissertations, and Problem Reports. 10267.

https://researchrepository.wvu.edu/etd/10267

This Problem/Project Report is protected by copyright and/or related rights. It has been brought to you by the The Research Repository @WVU with permission from the rights-holder(s). You are free to use this Problem/Project Report in any way that is permitted by the copyright and related rights legislation that applies to your use. For other uses you must obtain permission from the rights-holder(s) directly, unless additional rights are indicated by a Creative Commons license in the record and/ or on the work itself. This Problem/Project Report has been accepted for inclusion in WVU Graduate Theses, Dissertations, and Problem Reports collection by an authorized administrator of The Research Repository @ WVU. For more information, please contact researchrepository@mail.wvu.edu. 


\section{Re-design of Precision Signal Conditioning Circuit for detecting Schumann Resonance}

\section{Rohith Bikkina}

Problem Report submitted to the

Benjamin M. Statler College of Engineering and Mineral Resources

at West Virginia University

In partial fulfillment of the requirements

for the degree of Master of Science

in

Electrical Engineering

Roy S. Nutter, Jr., Ph.D., Chair

Brian D. Woerner, Ph.D.

Mohamed Hefeida, Ph.D.

Lane Department of Computer Science and Electrical Engineering

West Virginia University

Morgantown, West Virginia

2021

Keywords: Schumann Resonance, Amplifier, Low Pass Filter, PCB

Copyright 2021 Rohith Bikkina 


\title{
ABSTRACT \\ Re-design of Precision Signal Conditioning Circuit for detecting Schumann Resonance
}

\begin{abstract}
Rohith Bikkina
Extremely low frequencies signals are waves between 3 to $30 \mathrm{~Hz}$ and corresponding wave lengths between 10,000 to 100,000 kilometers. The specific signals used here are generated from lightning and are excited at frequencies around $8 \mathrm{~Hz}, 14 \mathrm{~Hz}, 20 \mathrm{~Hz}$. These are often called Schumann Resonance frequencies. Several stations have been built around the world for identifying ELF waves. All of those required a sparsely populated area that was far away from electric power lines because of interference from electric noise at $50 \mathrm{~Hz}$ and $60 \mathrm{~Hz}$. This project develops and tests an amplifier and filter circuit that should assist in identifying the Schumann Resonance signals in more electrically noisy environments. Detecting these extremely small signals requires circuits which have low internal noise among other characteristics. Analog Devices has recently produced a Precision Signal Conditioning Integrated Circuit (the AD8421) which has very low internal noise. The work reported herein uses Analog Devices new chip and amplifies these very low voltages and then actively filters electric noise at these low frequencies using the AD8510. A Printed Circuit Board (PCB) was designed to help shield the circuit from interference of higher frequencies in surrounding environment. This design detects input voltages below $5 \mathrm{uV}$ and gives a significantly amplified output. Voltage vs frequency and input vs output voltage characteristics are plotted for this design.
\end{abstract}




\section{Acknowledgements}

Foremost, I would like to express my sincere gratitude to my Committee Chair and advisor, Dr. Roy S. Nutter for his invaluable assistance and constant support throughout my time at WVU. He has been an incredible source of support and motivation. His guidance helped me in all the time work and in writing this document.

Apart from my advisor, I would like to acknowledge and thank my Problem Report committee members Dr. Brian Woerner and Dr. Mohamed Hefeida for their time and consideration.

I am also thankful to Dr. Matthew Valenti for providing me an opportunity to work as Graduate Services Assistant at LCSEE, WVU. I would also like to thank the LCSEE department for their support and opportunity.

Last but not least, it gives me immense pleasure to thank my family without whose support, this would not be possible. 


\section{Table of Contents}

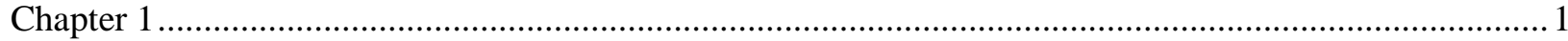

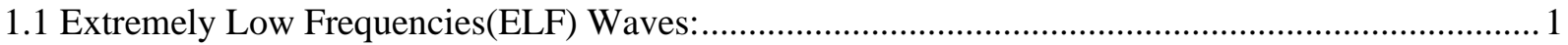

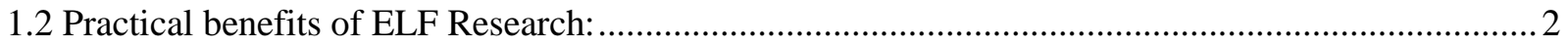

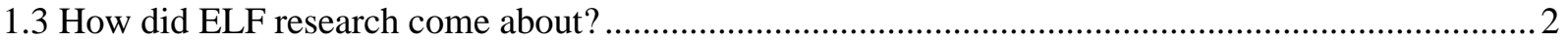

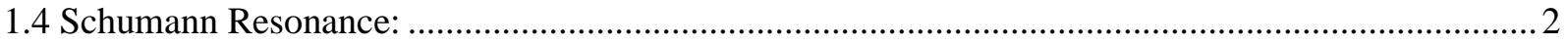

1.5 Observation of Schumann resonances at Hylaty station: .............................................................. 3

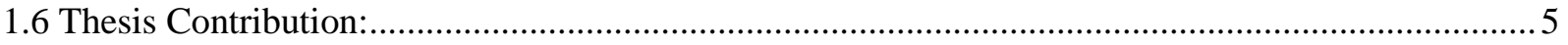

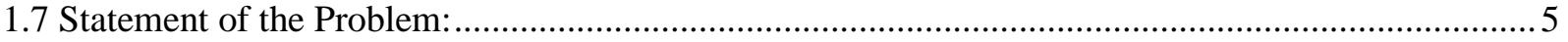

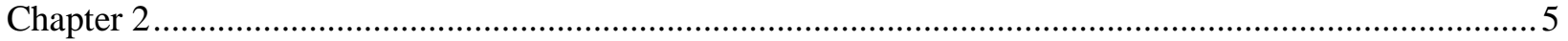

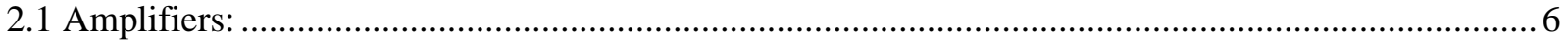

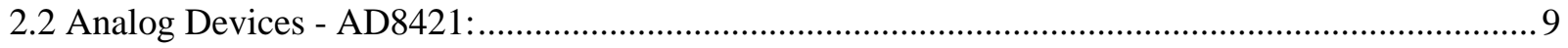

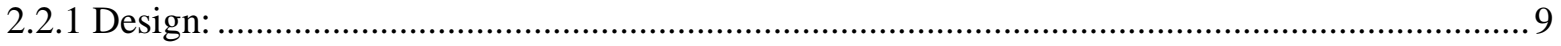

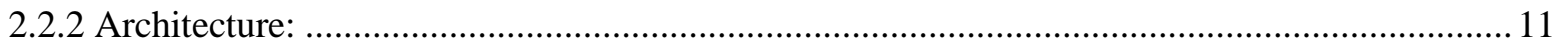

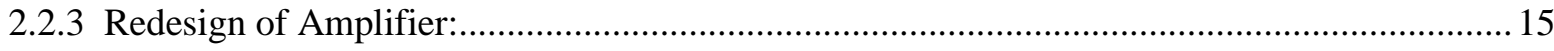

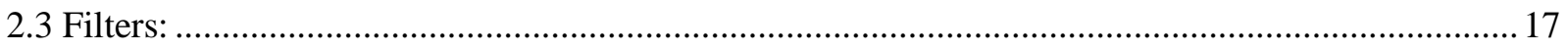

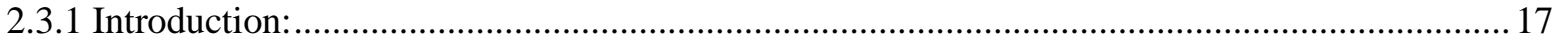

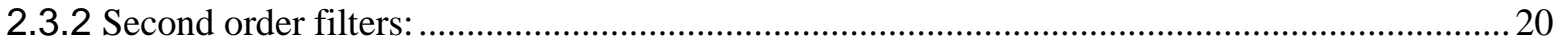

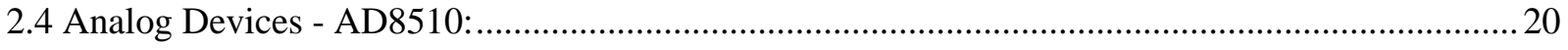

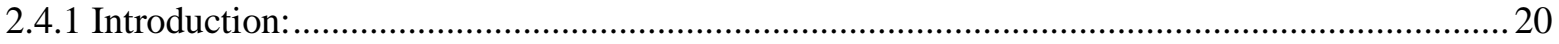

2.4.2 Example Filter circuit in Analog Devices (AN-1264) specification sheet:..............................22

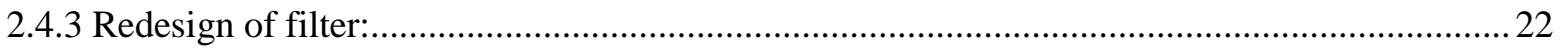

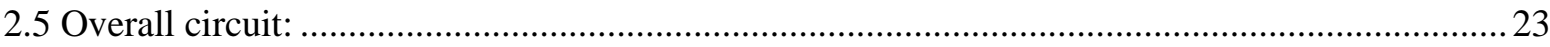

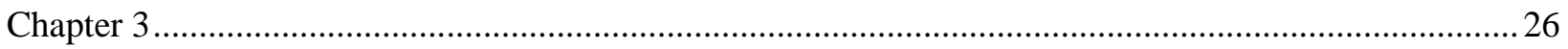

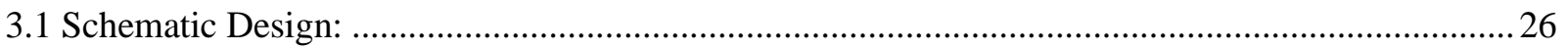

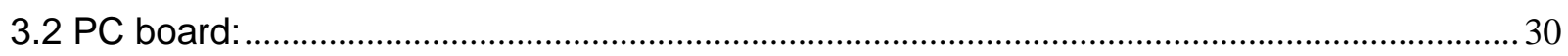

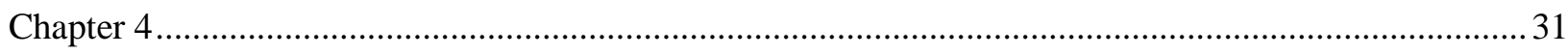

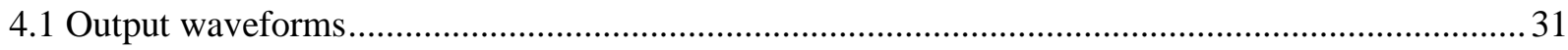

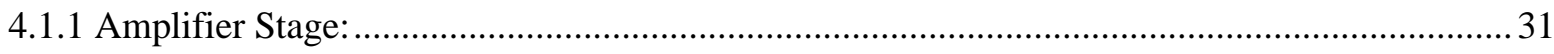

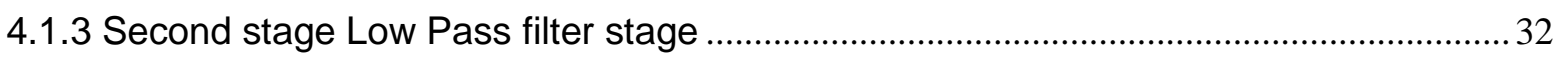

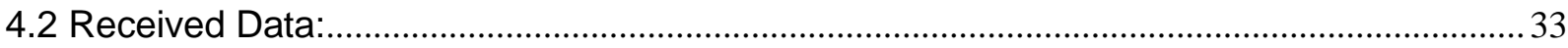

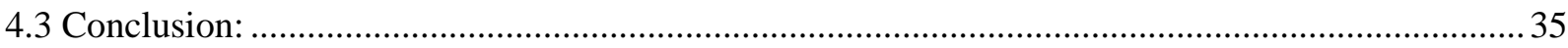

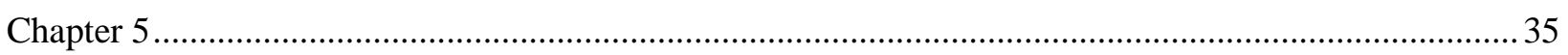

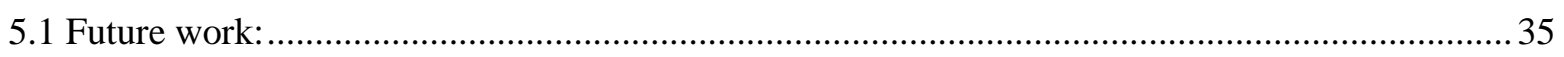

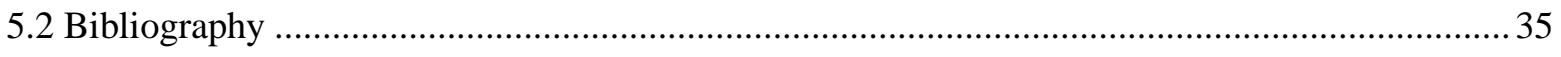




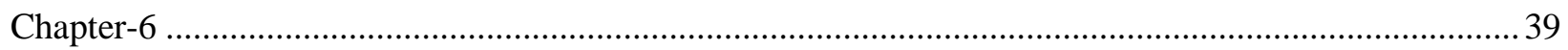

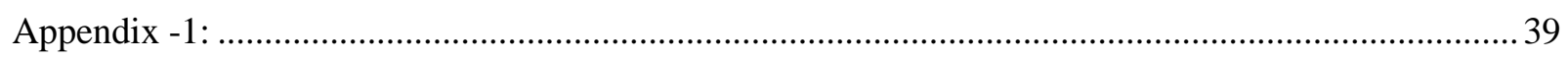

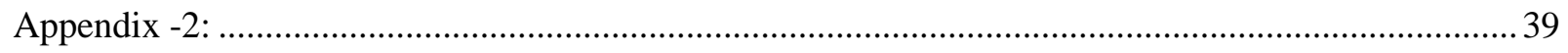




\section{List of Figures:}

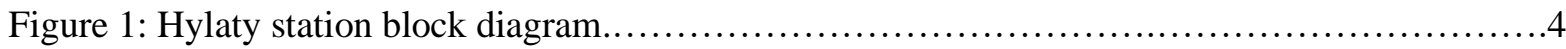

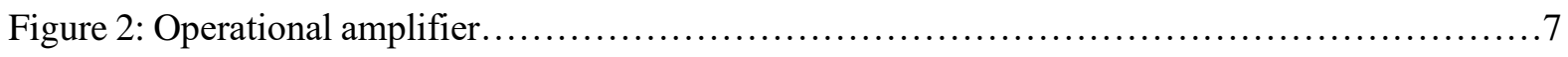

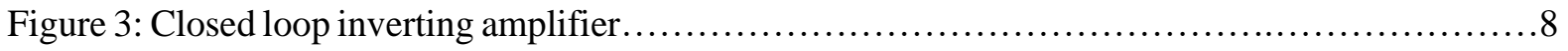

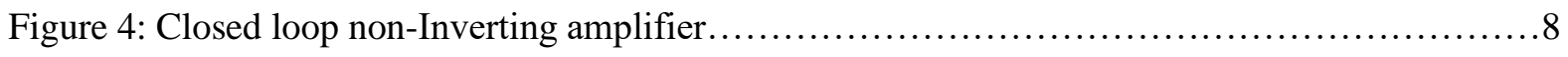

Figure 5: instrumentation amplifier design model $\ldots \ldots \ldots \ldots \ldots \ldots \ldots \ldots \ldots \ldots \ldots \ldots \ldots \ldots \ldots \ldots \ldots$

Figure 6: Source Resistance vs Noise Density for AD8421 .................................. 10

Figure 7: AD8421 Architecture.............................................................. 11

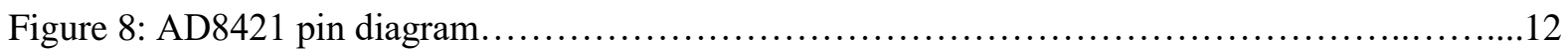

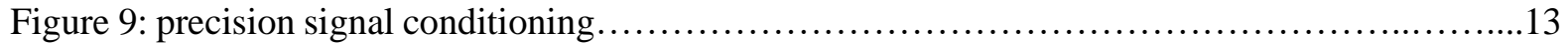

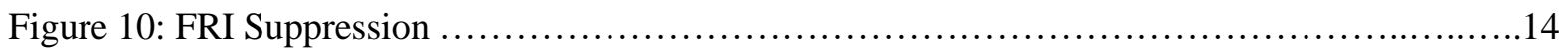

Figure 11: Low noise instrumentation amplifier by analog devices using AD8421.................15

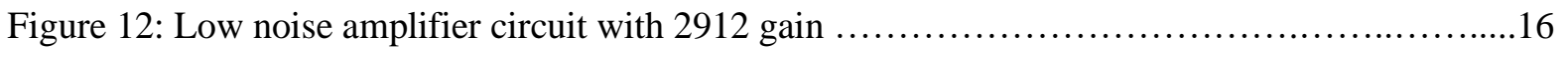

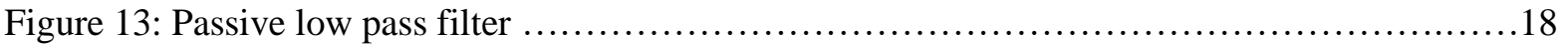

Figure 14: Active Low Pass Filter ....................................................... 19

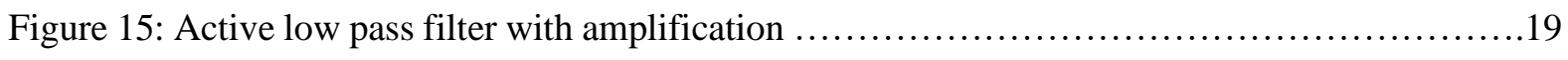

Figure 16: Sallen-Key Low Pass filter....................................................

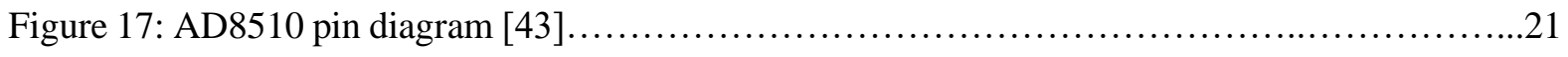

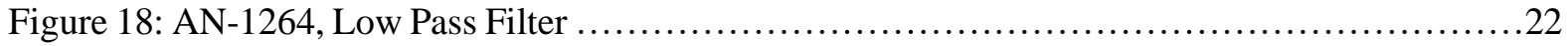

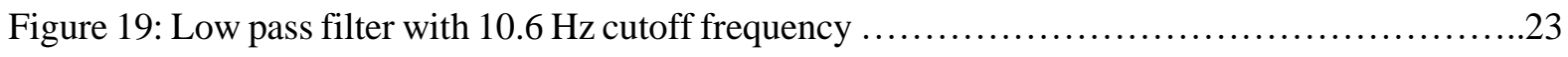

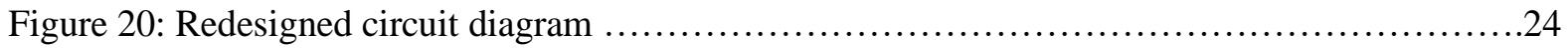

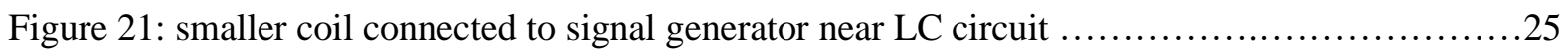

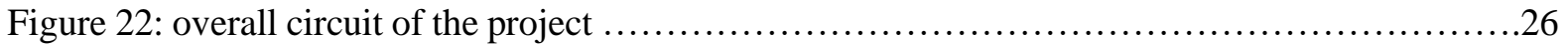

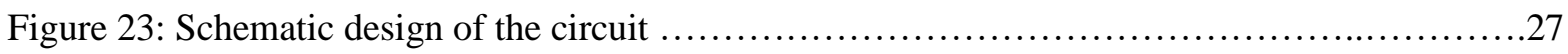

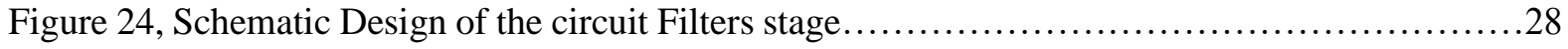

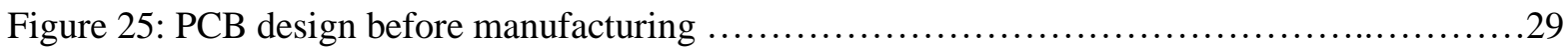

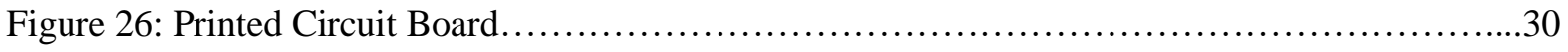

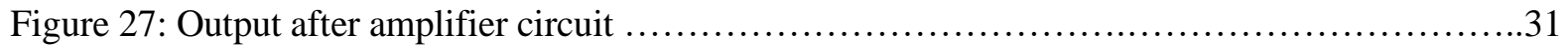




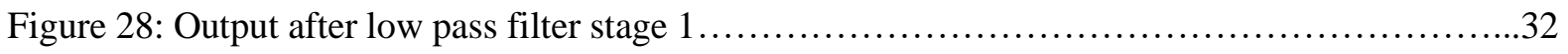

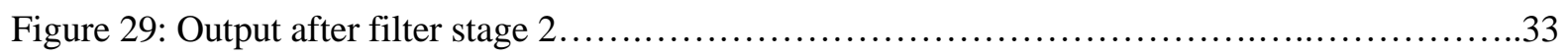

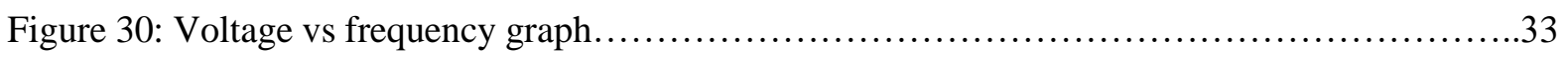

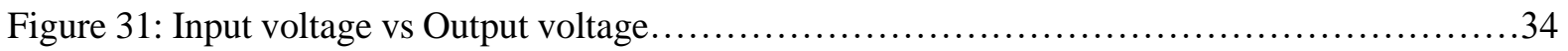

Figure 32: Input voltage range $(1.73 \mathrm{uV}-350 \mathrm{uV})$ vs Output voltage range $(5 \mathrm{mV}-1.02 \mathrm{~V}) \ldots \ldots \ldots . .34$

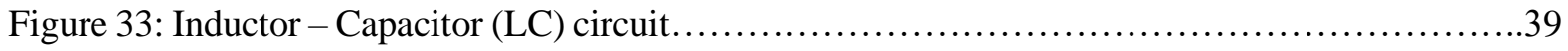

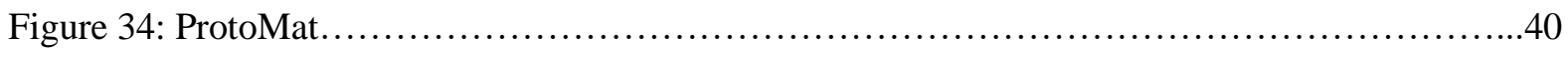

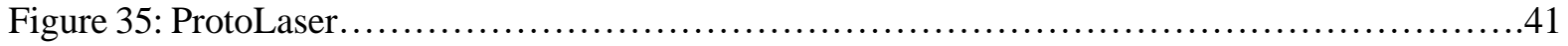




\section{Chapter 1 Introduction:}

\subsection{Extremely Low Frequencies (ELF) Waves:}

Signals at extremely low frequencies range in frequency from 3 to $30 \mathrm{~Hz}$ and in length from 10000 to 100000 kilometers [1]. The Schumann resonances are a set of electromagnetic resonances, generated by lightning discharges in the cavity formed by the Earth's surface and the ionosphere. They occur in the extremely low frequency (ELF) band between 6 and $50 \mathrm{~Hz}$ [2]. These signals have wavelengths of the order of earth's diameter and to transmit these signals earth itself should be used as an antenna [3]. As power lines emit ELF frequencies between $50-60 \mathrm{~Hz}$, according to Feychting and Ahlbom's epidemiological studies over 100000 Swedish children between 1960 and 1985 , if the distance from $200 \mathrm{kV}$ and $400 \mathrm{kV}$ power lines is less than 50 meters, the risk of developing leukemia is 2.9 [4]. ELF waves penetrate to depths of hundreds of feet in the sea [5]. Naval Computer and Telecommunications Area Master Station - Atlantic operates two extremely low frequency radio transmitters to communicate with deep diving submarines. The sites are located at Clam Lake, Wisconsin and Republic, Michigan [6].

Scientifically, ELF waves are very useful because they are mostly reflected in the $D$ region of the earth's ionosphere $(60-90 \mathrm{~km}$ altitude), and are therefore efficiently guided in the earth's ionosphere, waveguide to global distances. If you set up a radio receiver on earth, you can pick short bits of radiation from lightning strikes around the world. These are called atmospherics or sferics. ELF/VLF reflectivity at the $D$ region is a unique tool for remote sensing of the $D$ region. It responds to a variety of inputs like solar activity, lightning energy, electron precipitation from the radiation belts, cosmic gamma-rays, and earthquakes [11]. These measurements are extremely challenging because the altitudes of the $D$ region are too high to be measured by balloons and too low to be measured by satellites, so this is a useful ability. However, these studies are complicated by the fact that radio waves propagation in complex media is one of the most fundamentally difficult in electromagnetism, violating nearly every convenient simplifying assumption including homogeneity, linearity, symmetry, and anisotropy.

Insects are affected by ELF frequencies in a variety of ways. Locusts entrained to the precise frequency of the applied EMF after being exposed to a variety of ELF EMFs that were near to typical wingbeat frequency. In locusts, exposure to ELF EMFs caused tiny but substantial alterations in wingbeat frequency [7]. Clinical implications of ELFEMF are possible for the improvement of neurodegenerative processes and in the development of regenerative medicine [8]. 


\subsection{Practical benefits of ELF Research: Global Communications:}

Electromagnetic waves below $100 \mathrm{~Hz}$ penetrate far into ocean, making ELF suitable for communication with submarines. The waveguide generated between the earth and the ionosphere is used to propagate these frequencies [9].

\section{Lightning Geolocation:}

Based on ELF readings from a single station, ELF is utilized for worldwide localization of severe lightning strokes linked with Transient Luminous Events [53].

\subsection{How did ELF research come about?}

ELF/VLF observation began in the late 1800 s with the installation of the first telegraph wires. The early operators reported hearing weird sounds they described as clicks and tweeks, particularly on days when lightning struck, which we now know were most likely sferics picked up by their telegraph line, which served as an antenna.

Later in World War I, soldiers handling communication links reported hearing unusual tones that sounded like someone was whistling. "You can hear the grenades falling," some used to say. What they were actually hearing was an indirect form of lightning energy, called whistler now, coupling into the communication lines [11].

\subsection{Schumann Resonance:}

When an object is free to vibrate, it tends to do so at a specific rate termed the natural or resonant frequency of the object. When this object is subjected to vibrations or regular impulses at a frequency extremely near to its natural frequency, it will vibrate strongly. This is referred to as resonance [12].

At any given time, over 2000 thunderstorms sweep across the globe, producing approximately 50 flashes of lightning each second. Each lightning strike generates electromagnetic waves that begin to orbit the Earth, trapped between the Earth's surface and a 60-mile-high limit. Certain waves - assuming they have the precise wavelength - combine to form a recurring atmospheric heartbeat known as Schumann resonance. This resonance is a valuable phenomenon for analyzing the Earth's weather and electric environment, as well as for determining the sorts of atoms and molecules that exist in the Earth's atmosphere [13]. WO Schumann anticipated these waves for the first time in 1952 [14]. Certain frequencies of waves created by lightning travel through the Earth's surface and become intensified at around 8, 14, 20, and $26 \mathrm{~Hz}$ (the first, 
second, third, and fourth harmonics), which is referred to as Schumann resonance (SR). Koing discovered Schumann resonances for the first time experimentally by examining noise waveforms at the output of a narrow band amplifier [15][16][17]. Balser and Wagner [18] were the first to get detailed frequency spectra of this noise. Fournier [19]; Benoit and Houri [20,21]; Polk and Fitchen [22]; Gendrin and Stefant [23]; Balser and Wagner [24]; Rycroft [25]; Chapman and Jones [26] have all reported further measurements. ELF research saw a renaissance in the 1990s, owing mostly to its potential uses in climate studies [27]. Following then, numerous more stations were constructed in Hungary [28], Poland, Israel [29], and Japan [30] and more.

\subsection{Observation of Schumann resonances at Hylaty station:}

ELF waves were first recorded in a systematic fashion at Jagiellonian University in 1994 in Krakow, Poland. At first, measurements were made infrequently on expeditions to sparsely populated areas of Poland's Bieszczady Mountains. Continuous recording of electromagnetic fields below $60 \mathrm{~Hz}$ was accomplished in 2004 when the Hylaty ELF station was erected, allowing for continuous recording of the magnetic field components of the ELF electromagnetic field below $60 \mathrm{~Hz}$. In 2013, the station's frequency range was increased to $300 \mathrm{~Hz}$ [31].

It is necessary to observe natural electromagnetic fields in the range of $0.03-300 \mathrm{~Hz}$ with very low levels of man-made noise, and only a small number of power lines should be located near the station, with a population density that does not exceed a few people per square kilometer, to conduct this research. By changing Earth's magnetic fields, vibrating ferromagnetic items such as iron components from buildings may also produce noise by modulating the Earth's magnetic fields. In order to avoid being too close to major metropolitan or industrial hubs, preferred distances are several hundred kilometers distant. The Jagiellonian University's Hylaty geophysical station is in the Bieszczady Mountains, adjacent to the settlement of Zatwarnica, in the southeast of Poland, near the border with Ukraine. Located on the boundary of Bieszczady National Park, the station was named after a nearby stream known as Hylaty. It is in an uninhabited location, distant from the electrical power system, and on the outskirts of the park. There are currently two receivers installed in an underground container covered with a 1 meter deep ground layer at the geographic coordinates 49.2035 degrees $\mathrm{N}$ and 22.5438 degrees $\mathrm{E}$. The station is located on a gentle slope of a hill at the geographic coordinates 49.2035 degrees $\mathrm{N}$ and 22.99 degrees $\mathrm{E}$. Containers constructed of epoxy laminate, which have maintained a temperature of 2 degrees Celsius throughout the course of seven years. It contains two receivers that are synced with the GPS clock, as well as a power source that allows for two months of continuous operation without the need to recharge. To avoid electromagnetic interference, the magnetic antenna of the station, as well as their preamplifiers, are situated 100 meters 
distant from the receiver, which effectively eliminates any electromagnetic interference. In 2013, a second set of antennas was deployed, this time with a greater bandwidth capability. At a shallow depth below ground level, antennas are enclosed in a hermetic cage to maintain their integrity. With the station, the antennas are connected using hermetic pipes. A maximum noise amplitude of $10 \mathrm{pT}$ at $50 \mathrm{H}$ is seen at the Hylaty station [31].

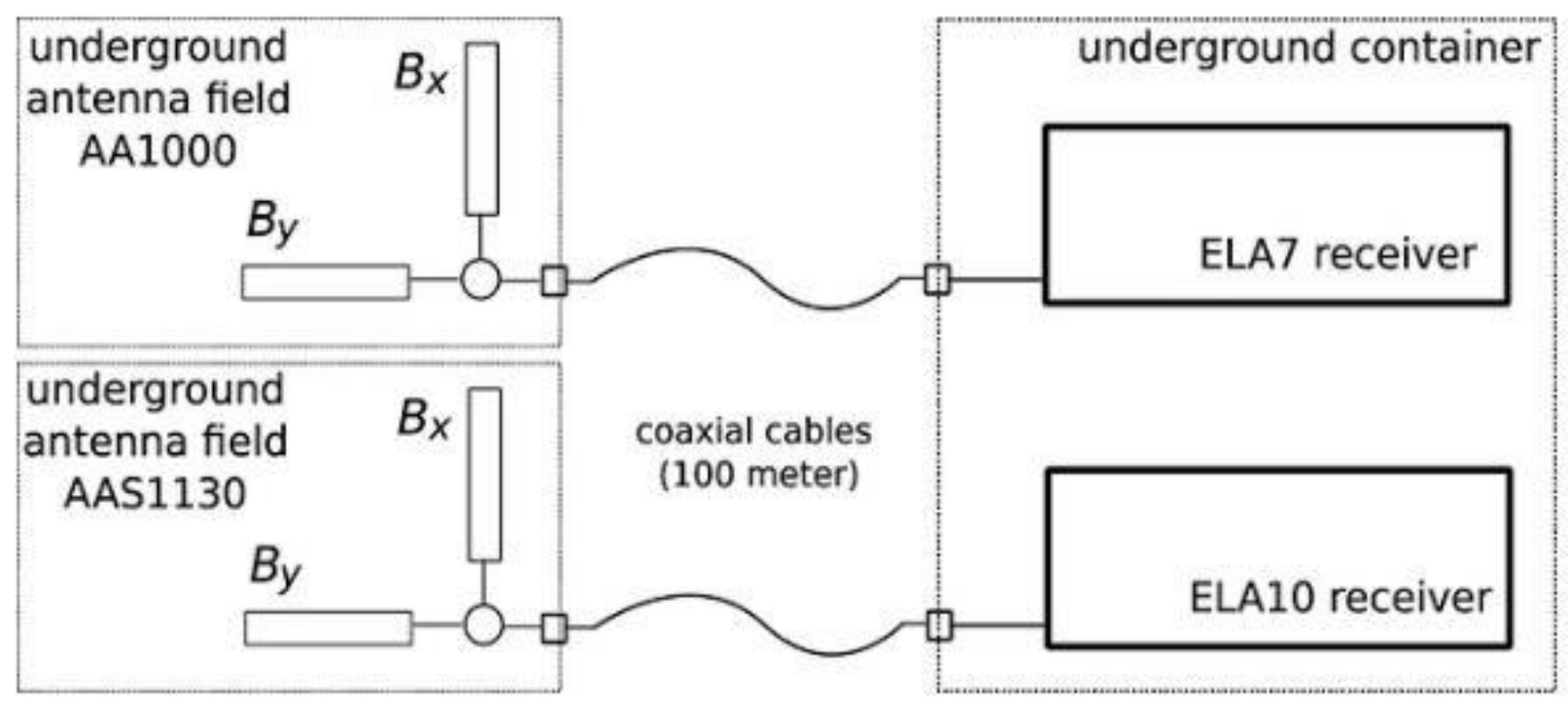

Figure 1, Hylaty station block diagram [31]

To function effectively, electric antennas require an unobstructed space. Otherwise, surrounding objects reduce the amplitude of the recorded signal and create noise. Due to the fact that the antenna field at the Hylaty station is located in a wooded area, only intermittent measurements of the electric field component are done using an expeditionary antenna. The output voltage of an induction coil is proportional to the rate at which the magnetic flux changes, $\mathrm{dB} / \mathrm{dt}$ (Faraday law). As a result, its sensitivity improves as frequency increases (at a rate of $6 \mathrm{~dB} /$ octave). Different antennas with low noise levels such as the AB1000, AB600, AA1000, AA1130, AA1500, and AAS1130 were created in the years 1993, 1997, 2003, 2009, 2010, and 2012, respectively.

In 1994, the first battery-powered digital receiver was created, where "ELA" stands for the receiver's name and is followed by the version number. The receiver was fitted with a 12-bit analog-to-digital converter (ADC) and connected via a 5-meter coaxial cable to a passive antenna, AB1000. ELA2 was intended as the next generation receiver in 1997. It included four measurement channels, three sensitivities, and was powered by an integrated $12 \mathrm{~V} / 12 \mathrm{~A} \mathrm{~h}$ gel battery. It permitted the activation of low-pass filters with a bandwidth of $20 \mathrm{~Hz}, 40 \mathrm{~Hz}$, or $60 \mathrm{~Hz}$. The next generation of ELA3, ELA4, and ELA5 prototypes can hold data up to several gigabytes. 
At the output of an active antenna, the Schumann resonance background produces signals that do not surpass few microvolts. These pulses are powerful enough to be sent across a coaxial wire to a receiver several hundred meters away. Since 2006, the ELA7 has been the primary kind of receiver utilized at the Hylaty Station. It is a fourchannel, 16-bit receiver with a bandwidth of 0.03 to $52 \mathrm{~Hz}$ and a flash memory for data storage. The receiver is powered by a $12 \mathrm{~V} / 260$ Ah battery and can operate without human intervention for two months. The great precision of sampling time necessitates the use of a very stable clock generator that is modified using an external time reference. Specialized GPS time reference circuits (GPS disciplined clock) might be used for this purpose; however, they use a few watts of power.

The ELA7 and ELA10 receivers operate on a few hundred milliwatts and are powered by separate sets of $12 \mathrm{~V}$ batteries with a capacity of 260 -amp hours. They enable the station to operate continuously for more than two months. The operator charges the batteries through a power generator [31].

\subsection{Thesis Contribution:}

- Redesign of the Precision Signal Conditioning for High Resolution Industrial Applications [32] circuit with appropriate cut off frequency and appropriate damping factor to obtain a clear and plain sine wave.

- Designing a printed circuit board, PCB, to improve the noise environment for the circuit.

- Characterize this design.

\subsection{Statement of the Problem:}

This work redesigns and characterizes Precision Signal Conditioning circuit for the Schumann Resonance frequencies.

\section{Chapter 2}

\section{Background of Re-design:}




\subsection{Amplifiers:}

An amplifier is a type of electrical device that boosts the strength of a signal. An amplifier's purpose is to boost the power of a signal without distorting it. This is referred to as Amplification. For instance, to magnify the control signals generated by an aircraft's pilot in order for them to move the flaps on the wings and other control devices. [32]

Operational amplifiers are a type of amplifier that serve as the foundation for audio and video amplifiers, filters, buffers, line drivers, instrumentation amplifiers, comparators, and oscillators, amongst other analogue circuits. Operational amplifiers are frequently abbreviated as op-amps. [33]

Op-amps typically have two inputs, which are referred to as the non-inverting input(-) and the inverting input(+). Usually op-amps have single output, but special op-amps used in radio frequency circuits have two outputs [34].

An operational amplifier (op-amp) is a differential amplifier. its output is proportional to the difference between the two input signals. If the positive input is at a greater potential than the negative input, the output is positive, if the negative input is at a lower potential than the positive input, the output is negative. The output will be the product of the difference between the two inputs multiplied by the gain. Gain for operational amplifiers is typically about 100000 . This phenomenon is referred to as open loop gain. Additionally, the op-amp is coupled in a closed loop mode, where gain is controlled and always lower than the open loop gain [33].

\section{Op-amp parameter and idealistic characteristics:}

Open Loop Gain():

The gain of an op-amp without positive or negative feedback is limitless, however common practical values vary from 20,000 to 200,000 .

Input Impedance(Zin):

The input impedance is defined as the ratio of the input voltage to the input current and is assumed to be infinite to prevent any current from the source supply flowing into the amplifier's input circuitry.

Output impedance (Zout):

The output impedance of an ideal op-amp is zero, allowing it to operate as a perfect internal voltage source with no internal resistance, supplying the load with the maximum current possible. Real op-amps have an output impedance of between $100 \mathrm{ohms}$ and 20 kilo ohms.

Bandwidth(BW): 
Because an ideal operational amplifier has an unlimited frequency response and is capable of amplification of any frequency signal from $D C$ to the highest $A C$ frequencies, it is believed to have an infinite bandwidth. The bandwidth of genuine op-amps is however restricted by the Gain-Bandwidth product (GB), which equals the frequency at which the amplifier's gain equals unity [35].

The ideal op-amp(open loop):

- Output depends only on the difference between voltages applied to the two input terminals.

- Performance is entirely dependent on input and feedback networks.

- No current flows into amplifier input terminals.

- Amplifier is unaffected by load.

- When the input signal voltage is zero, output is also zero.

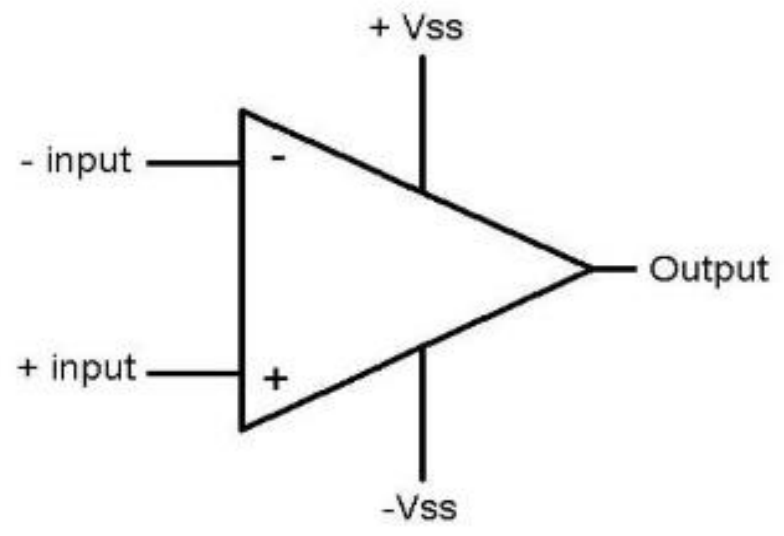

Figure 2, operational amplifier [40]

\section{Closed loop amplifier:}

Amplifiers in open loop configurations have extremely high gain, which is useless. Due to the amplifier's extremely high open loop DC gain, one may lower and regulate the amplifier's overall gain by connecting a resistor across the amplifier from the output terminal to the input terminal. Feedback can be applied to the amplifier's inverting and non-inverting input terminals to produce an inverting or non-inverting output signal.

Inverting Amplifier:

Negative feedback is the process of returning a portion of the output signal to the opinverting amp's terminal through an external feedback resistor R2 shown in Figure 3. It decreases the amplifier's total gain; this is referred to as closed loop gain. The noninverting terminal is grounded in this setup.

Closed loops gain of the inverting amplifier is, Gain $(A v)=-R 2 / R 1$. 


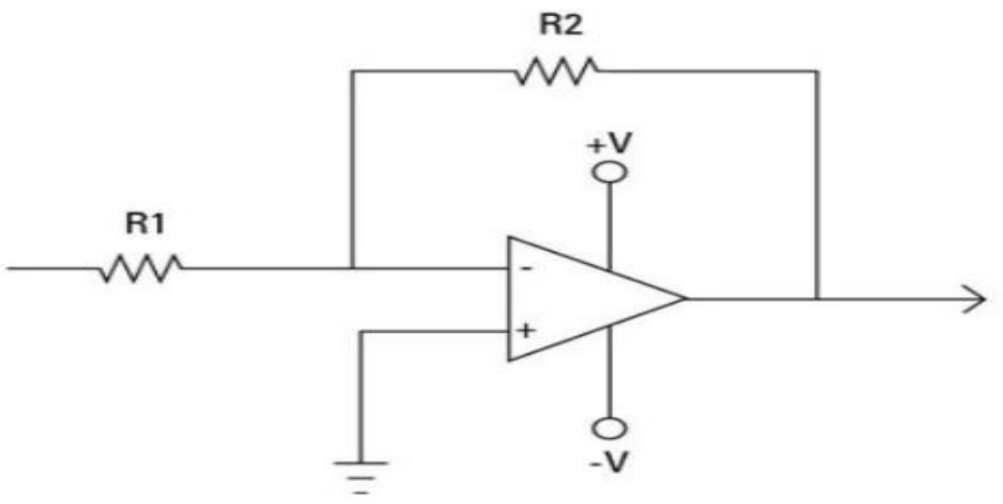

Figure 3, Closed loop inverting amplifier [41]

Non-inverting amplifier:

The Vin is applied directly to the non-inverting terminal in this setup, where the feedback and input signal are in phase. The non-inverting operational amplifier is controlled by negative feedback by applying a tiny portion of the output voltage signal back to the inverting input terminal via an R2 - R1 voltage divider network.

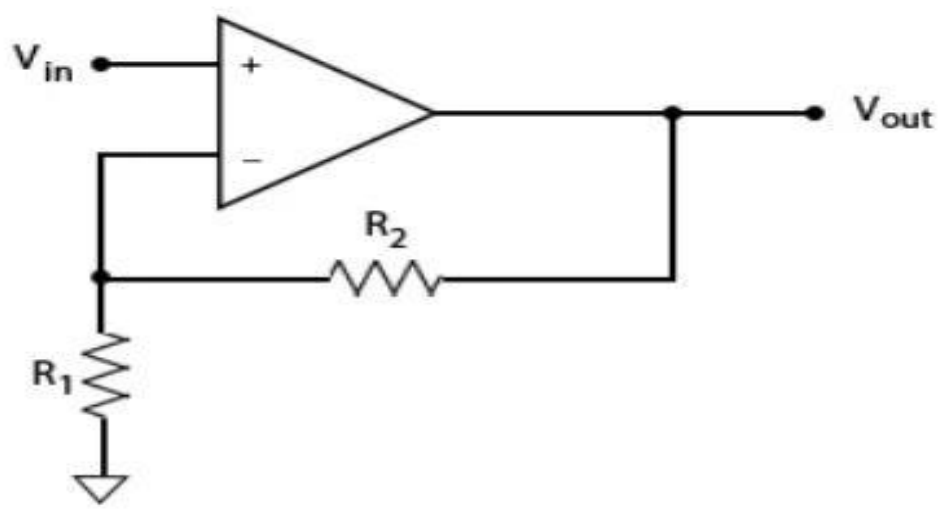

Figure 4, Closed loop non-Inverting amplifier [50]

This closed-loop architecture results in a non-inverting amplifier circuit with excellent stability, a very high input impedance, Rin, approaching infinity (ideal circumstances), and a low output impedance, Rout.

Closed loop gain of non-inverting amplifier $A v=$ Vout $/$ Vin $=1+R 2 / R 1$ [35].

\section{Feedback and Ideal op-map:}

- When negative feedback is applied to the ideal amplifier, the differential input voltage is zero.

- No current flows into either input terminal of the ideal amplifier. 
Instrumentation amplifier:

Instrumentation amplifiers are differential operational amplifiers that combine high impedances with the convenience of adjusting gain through a single resistor variation [36]. It is a differential amplifier with the addition of buffer stages at the inputs. By including input buffer stages, it becomes simple to match the amplifier to the previous stage. Amplifiers for instruments are often utilized in industrial test and measurement applications. Additionally, the instrumentation amplifier has several advantageous characteristics such as a low offset voltage, a high Common Mode Rejection Rati $\mathrm{o}(\mathrm{CMRR})$. The CMRR indicates the ability of a differential amplifier to suppress signals common to the two inputs [51].

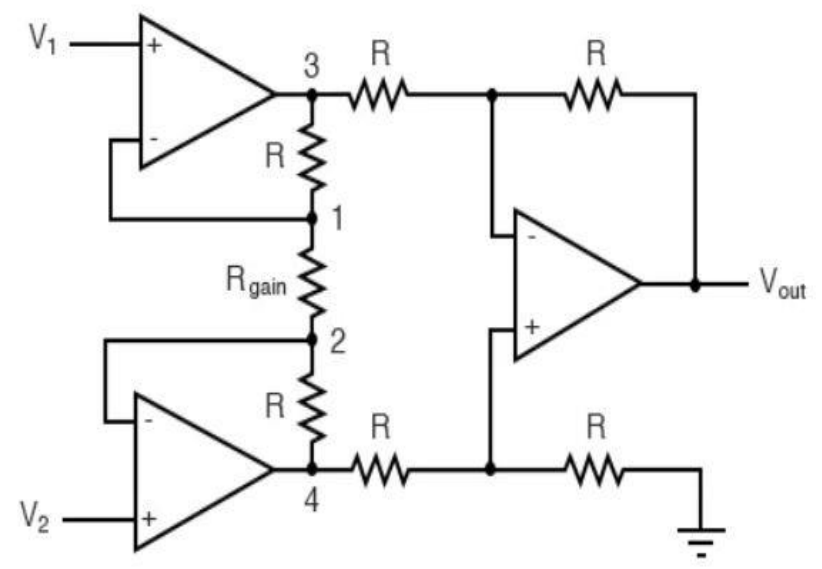

Figure 5, instrumentation amplifier design model [36]

Figure 5 represents an instrumentation amplifier circuit. For simplicity, if all the resistors in the circuit are considered the same. The op-amps on the left serve as input buffers for the differential amplifier on the right. Gain is also not unity on the buffers on the left side due to the existence of resistors $R$ and Rgain. The voltage gain of instrumentation amplifier is expressed with the equation

Voltage gain $(A v)=V o /(V 2-V 1)=(1+2 R / R g a i n)$

Gain of the amplifier can be changed by changing the resistor Rgain.

\subsection{Analog Devices - AD8421:}

\subsubsection{Design:}

The AD8421 is a low cost, low power, ultralow noise, ultralow bias current, high speed instrumentation amplifier that is well suited for a wide variety of signal conditioning and data gathering applications. This device has an extraordinarily high 
CMRR, which enables it to recover low-level signals in the face of high-frequency common-mode noise throughout a broad temperature range.

The AD8421's $10 \mathrm{MHz}$ bandwidth, $35 \mathrm{~V} / \mathrm{s}$ slew rate, and $0.6 \mathrm{~s}$ settling time to 0.001 percent $(G=10)$ enable it to amplify high-speed signals and excel in applications requiring multiplexed systems with a large channel count. Even with increased gains, the existing feedback design retains a high level of performance; for example, with $G=$ 100 , the bandwidth is $2 \mathrm{MHz}$ and the settling time is $0.8 \mathrm{~s}$. The AD8421's $10 \mathrm{MHz}$ bandwidth, $35 \mathrm{~V} / \mathrm{s}$ slew rate, and $0.6 \mathrm{~s}$ settling time to 0.001 percent $(G=10)$ enable it to amplify high-speed signals and excel in applications requiring multiplexed systems with a large channel count. Even with increased gains, the existing feedback design retains a high level of performance; for example, with $\mathrm{G}=100$, the bandwidth is $2 \mathrm{MHz}$ and the settling time is $0.8 \mathrm{~s}$. A single resistor adjusts the gain from one to ten thousand. The AD8421 operates between $40^{\circ} \mathrm{C}$ and $+85^{\circ} \mathrm{C}$ in the eight-lead MSOP and SOIC packages, and between $40^{\circ} \mathrm{C}$ and $+125^{\circ} \mathrm{C}$ in the eight-lead LFCSP package. The AD8421 has an input voltage noise of $3 \mathrm{nV} / \mathrm{Hz}$ and a current noise of $200 \mathrm{fA} / \mathrm{Hz}$ with a quiescent current of just $2 \mathrm{~mA}$, making it a perfect option for monitoring low level signals [38].

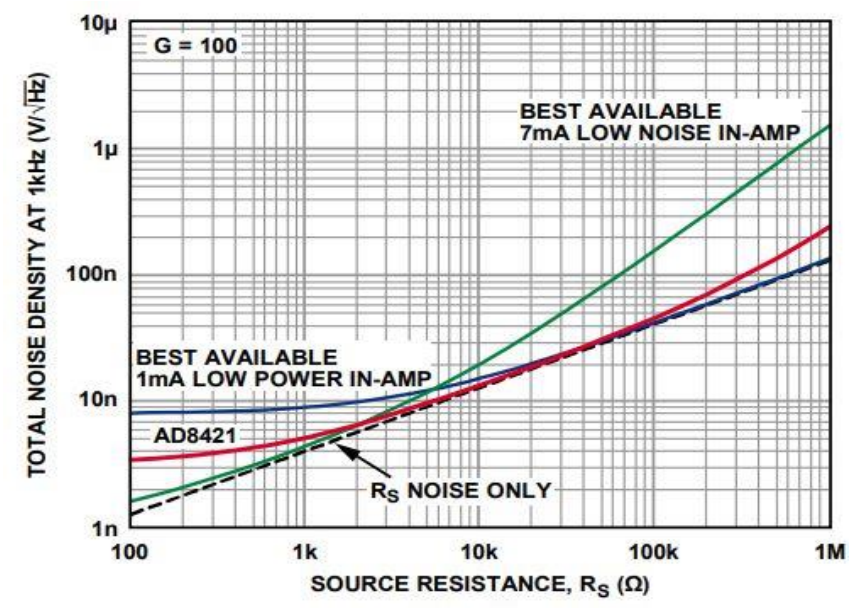

Figure 6, Source Resistance vs Noise Density for AD8421 [38]

AD8421 utilizes low power with a 2.3mA maximum quiescent current. . At a signal frequency of $20 \mathrm{KHz}$ and a gain of 1 , it has an $80 \mathrm{~dB}$ CMRR. The inputs are secured against reverse supply voltages of up to $40 \mathrm{~V}$. The input voltage ranges from $-2.5 \mathrm{~V}$ to +$18 \mathrm{~V}$ in dual supply mode and from $5 \mathrm{~V}$ to $36 \mathrm{~V}$ in single supply mode [38].

Due to its low noise characteristic, it finds a wide range of applications in medical instrumentation, precision data collection, microphone preamplification, vibration analysis, multiplexed input applications, and ADC drivers [38]. 


\subsubsection{Architecture:}

The AD8421 employs a standard three-op-amp architecture. This design consists of two stages: a preamplifier that performs differential amplification and a difference amplifier that eliminates common-mode voltage. Q1 and Q2 are biased at a constant current so that any input signal forces the output voltages of $A 1$ and $A 2$ to change accordingly. Across the RG pins, the differential signal applied to the inputs is repeated.

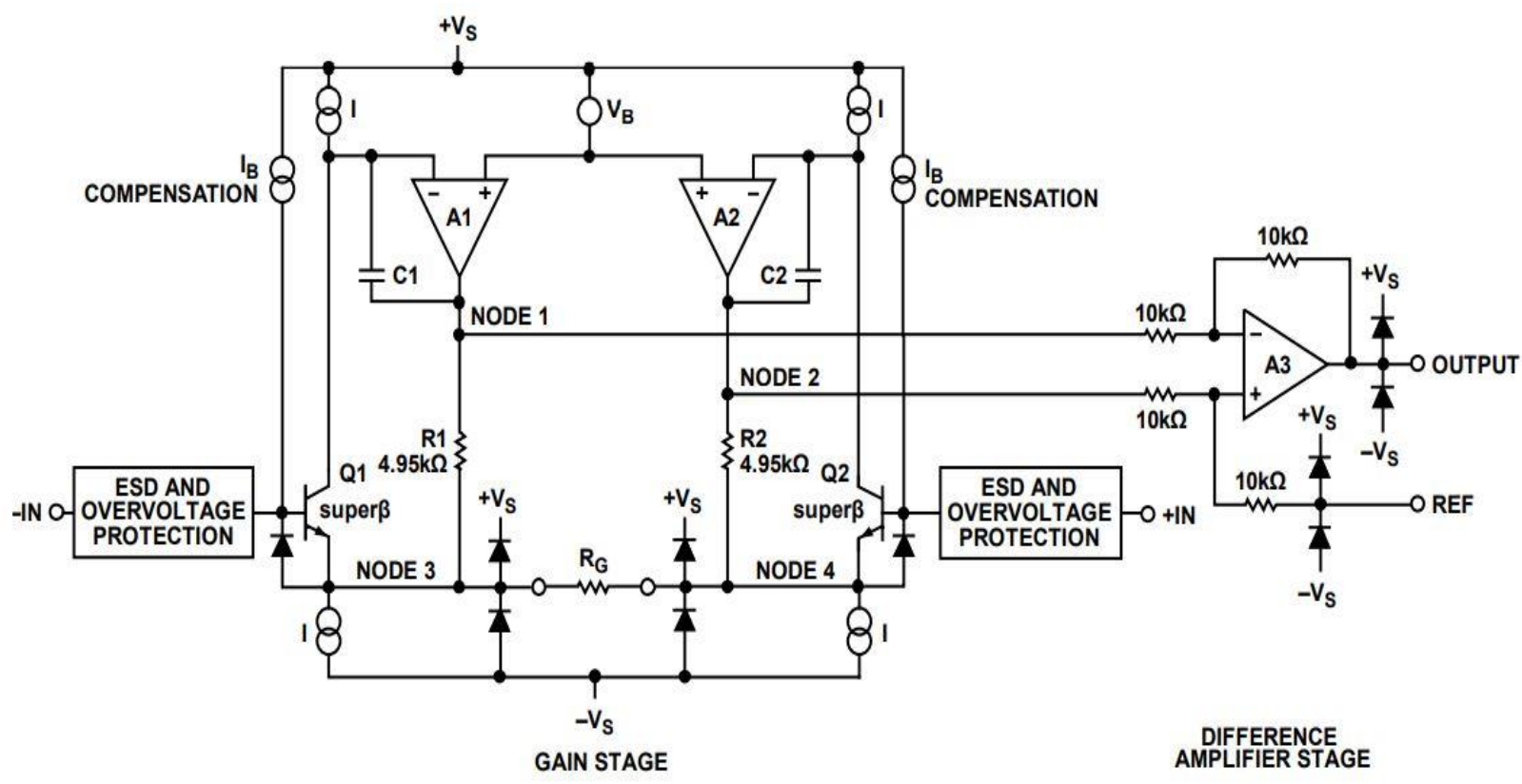

Figure 7, AD8421 Architecture [38]

Any current that passes through RG also passes through R1 and R2, resulting in a gained differential voltage between Nodes 1 and 2. "Laser-trimmed resistors enable the construction of a very precise in-amp with a gain error of less than 0.01 percent and a CMRR of greater than $94 \mathrm{~dB}(\mathrm{G}=1)$. The high-performance pinout and careful design and layout result in an extremely high CMRR performance over a wide frequency and temperature range" [38].

The AD8421 has exceptionally high input impedance, low bias current, low offset current, low current noise, and extremely low voltage noise of $3 \mathrm{nV} / \mathrm{Hz}$ by utilizing super beta input transistors and bias current compensation. The AD8421's transfer function is Vout $=G^{*}(V+$ in - V-in $)+$ Vref where $G=1+9.9 k O h m / R G$. [38]

\section{Reference Terminal:}

The AD8421's output voltage is developed in relation to the potential on the reference terminal. By connecting a voltage source to the REF pin, the output may be level shifted, allowing the AD8421 to drive a single supply ADC. The REF pin is 
protected by ESD diodes and should not exceed $0.3 \mathrm{~V}$ in either direction (+VS or -VS). [38]

Input Voltage Range:

The AD8421's three-op-amp design provides gain in the first stage and then eliminates the common-mode voltage in the difference amplifier stage. Internal nodes between the first and second stages are subjected to a mix of acquired, common-mode, and diode drop signals [38].

Layout:

The pins of the AD8421 are arranged in a logical manner. Pin diagram is shown in Figure 8.

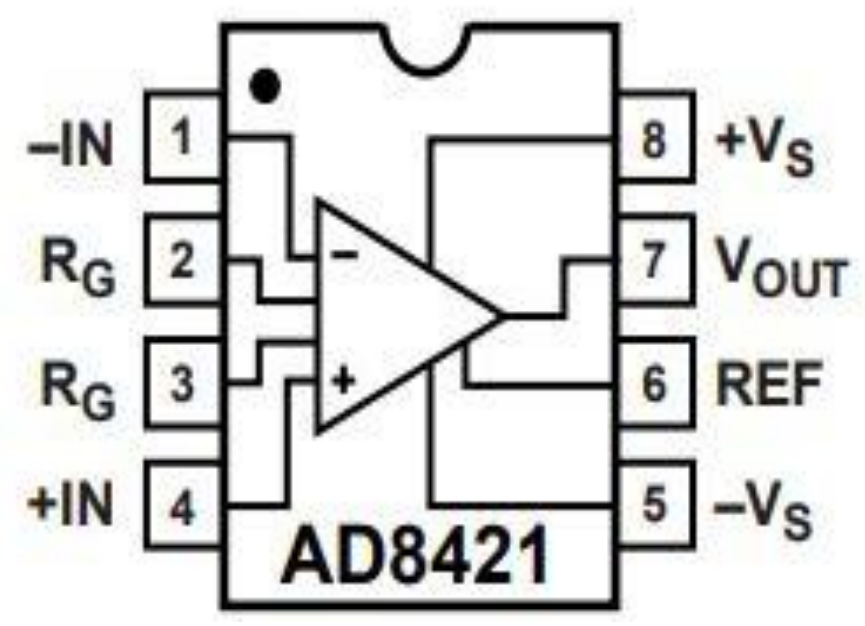

Figure $8, A D 8421$ pin diagram [38]

Common Mode rejection ratio over frequency:

To maintain a high CMRR across a broad frequency range, each path's input source impedance and capacitance should be tightly matched. Add extra source resistance to the input line near the in-amp inputs to limit the resistance's interaction with parasitic capacitance from the PCB traces [38].

Power supplies and grounding:

Place a $0.1 \mathrm{~F}$ capacitor as near to each supply pin as feasible. Due to the crucial nature of bypass capacitor lines at high frequencies, surface-mount capacitors are preferred. Any parasitic inductance in the bypass ground circuit works against the bypass capacitor's low impedance. As seen in Figure 9 , a 10uF capacitor may be employed at a greater distance from the device. The current return path distance is less crucial for these bigger value capacitors, which are meant to be effective at lower 
frequencies. A ground plane layer contributes to the reduction of parasitic inductances, hence minimizing voltage drops associated with current variations. Because load currents originate from the supply, the load should be physically linked to the bypass capacitor grounds [38].

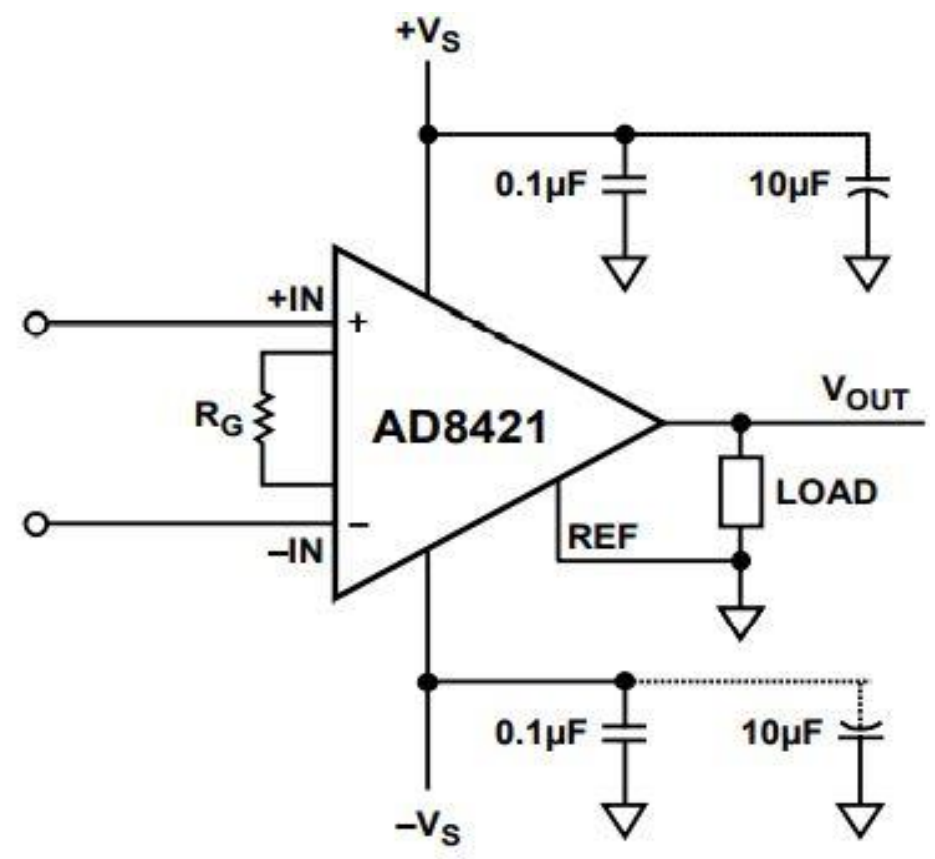

Figure 9, precision signal conditioning [38]

Reference pin:

The AD8421's output voltage is developed in relation to the potential on the reference terminal. Ascertain that REF is connected to the proper local ground [38]. Input Bias Current return path:

The AD8421's input bias current must have a route to ground when a floating source with no current return path is used in a circuit. Connecting $10 \mathrm{MOhms}$ resistors parallel to the inputs acts as a current return path to ground [38].

Input voltages beyond the supply rail:

The AD8421's inputs are quite robust. Generally, it does not require further input protection. Due to the fact that the AD8421 inputs are current restricted, input voltages from the opposing supply rail can be as high as $40 \mathrm{~V}$ [38].

Input voltages beyond the Maximum ratings: 
Use resistors in series with the inputs in conjunction with diodes for lengthier events. To minimize compromising bias current performance, it is advisable to use low leakage diodes such as the BAV199 or FJH1100. The diodes prevent the amplifier's input voltage from reaching its maximum rating, while the resistors restrict the current flowing through the diodes. Since the majority of external diodes are capable of handling 100 $\mathrm{mA}$ or more, resistor values do not need to be big and hence have a negligible effect on noise performance. At room temperature, the AD8421 inputs can tolerate a current of $40 \mathrm{~mA}$ for at least a day. This time is cumulative over the device's lifespan. If prolonged periods of overvoltage are anticipated, it is advisable to utilize an external protection technique. Under certain input conditions, the amplifier's output may reverse [38].

\section{Radio Frequency Interference:}

$\mathrm{RF}$ rectification is often a problem when amplifiers are used in applications that have strong RF signals. High frequency signals can be filtered with a low-pass filter network at the input of the instrumentation amplifier as shown in Figure 10.

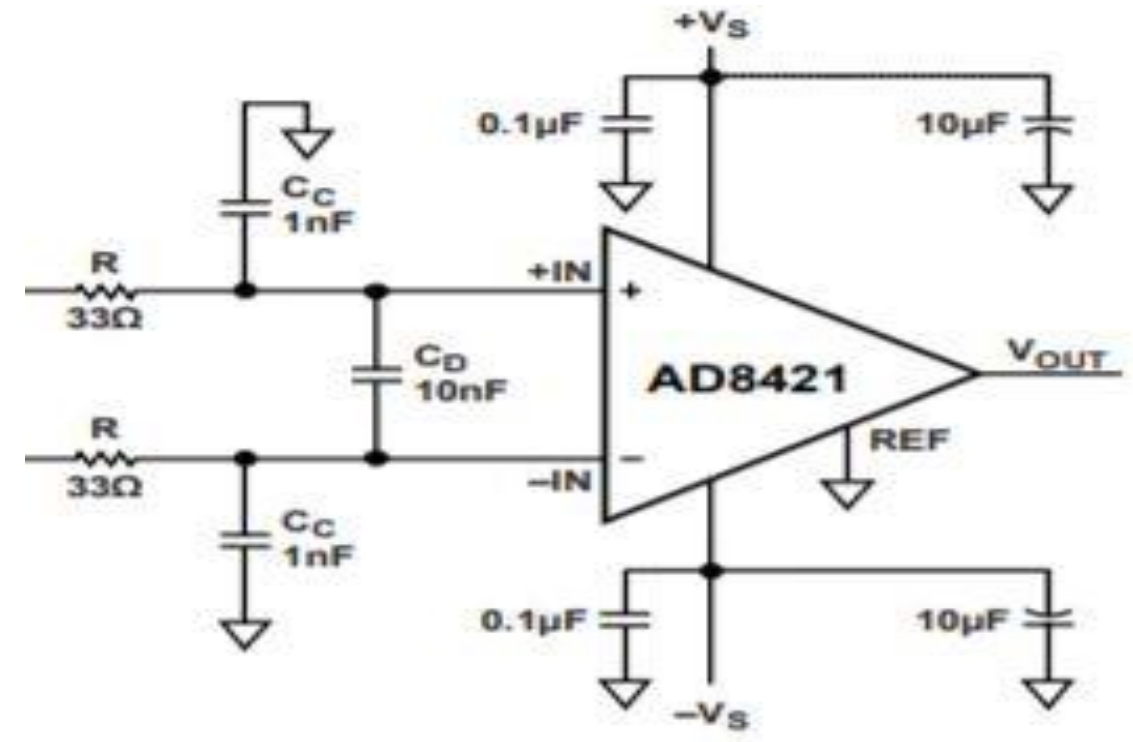

Figure 10, FRI Suppression

Filter Frequency $($ diff $)=1 / 2^{*} \mathrm{pi}^{*} \mathrm{R}(2 \mathrm{Cd}+\mathrm{Cc})$

Filter Frequency $(\mathrm{cm})=1 / 2^{*} \mathrm{pi}^{*} \mathrm{R}^{\star} \mathrm{Cc}$ where $\mathrm{Cd}>10 \mathrm{Cc}$

$\mathrm{Cd}$ has an effect on differential signals, while $\mathrm{Cc}$ has an effect on common mode signals. A mismatch between $\mathrm{R}^{\star} \mathrm{C} c$ at the positive and negative inputs weakens the AD8421's CMRR. By selecting a Cd value that is one order of magnitude more than Cc, the effect of the mismatch is mitigated and CMRR performance at the cutoff frequencies is enhanced.

Calculating the noise of input stage: 
The amplifier front end's total noise is highly dependent on factors other than the 3.2 $\mathrm{nV} / \mathrm{Hz}$ standard in the data sheet. The three major sources of noise are the source resistance, the instrumentation amplifier's voltage noise, and the instrumentation amplifier's current noise.

Analog Devices developed the AN1264 circuit utilizing the AD8421 because of AD8421's low noise characteristics. The amplifier that analogue devices used in AN1264 is seen in Figure 11. It is an active low pass instrumentation amplifier. In the circuit shown in Figure 11, 10MOhm resistors are connected to provide a conduit for the input bias current to reach ground. The circuit is protected from radio frequency interference by $4.7 \mathrm{Kohms}$ and $100 \mathrm{pF}$ capacitors. By utilizing $1 \mathrm{nF}$, the effect of mismatch is mitigated and the CMRR performance around the cutoff frequencies is enhanced. By adjusting a single resistor RG, the gain may be varied from 1 to 10000 .

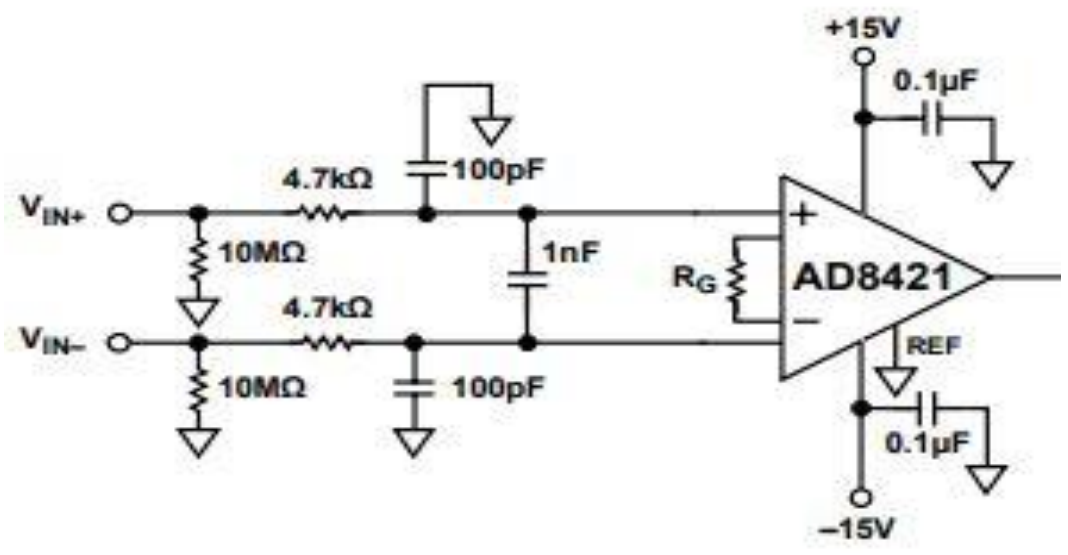

Figure 11, low noise instrumentation amplifier by analog devices using AD8421 [43]

In general, amplifier noise is composed of $1 / f$ noise and broad band noise. Typically, this noise affects low frequencies. When determining the overall noise contribution to an application, the operating bandwidth should be considered. The corner frequency is set to ten Hertz. When determining the overall noise contribution to an application, the operating bandwidth should be considered. The gain of the amplifier is determined by the equation $\mathrm{G}=1+(9.9 \mathrm{KOhm} / \mathrm{RG})$ [43].

\subsubsection{Redesign of Amplifier:}

A 3.4 Ohm resistor is used as $R G$ (gain resistor) to get a gain of 2912 , which significantly boosts the amplifier's gain. The image below illustrates the modified 
amplifier circuit. The amplifier's output is used as the filter's input. The amplifier's supply voltages are $+9 \mathrm{~V}$ and $-9 \mathrm{~V}$ at pins 8 and 5 , respectively. Additionally, 10uF capacitors are added to the circuit to protect the circuit from any fluctuations in the voltage.

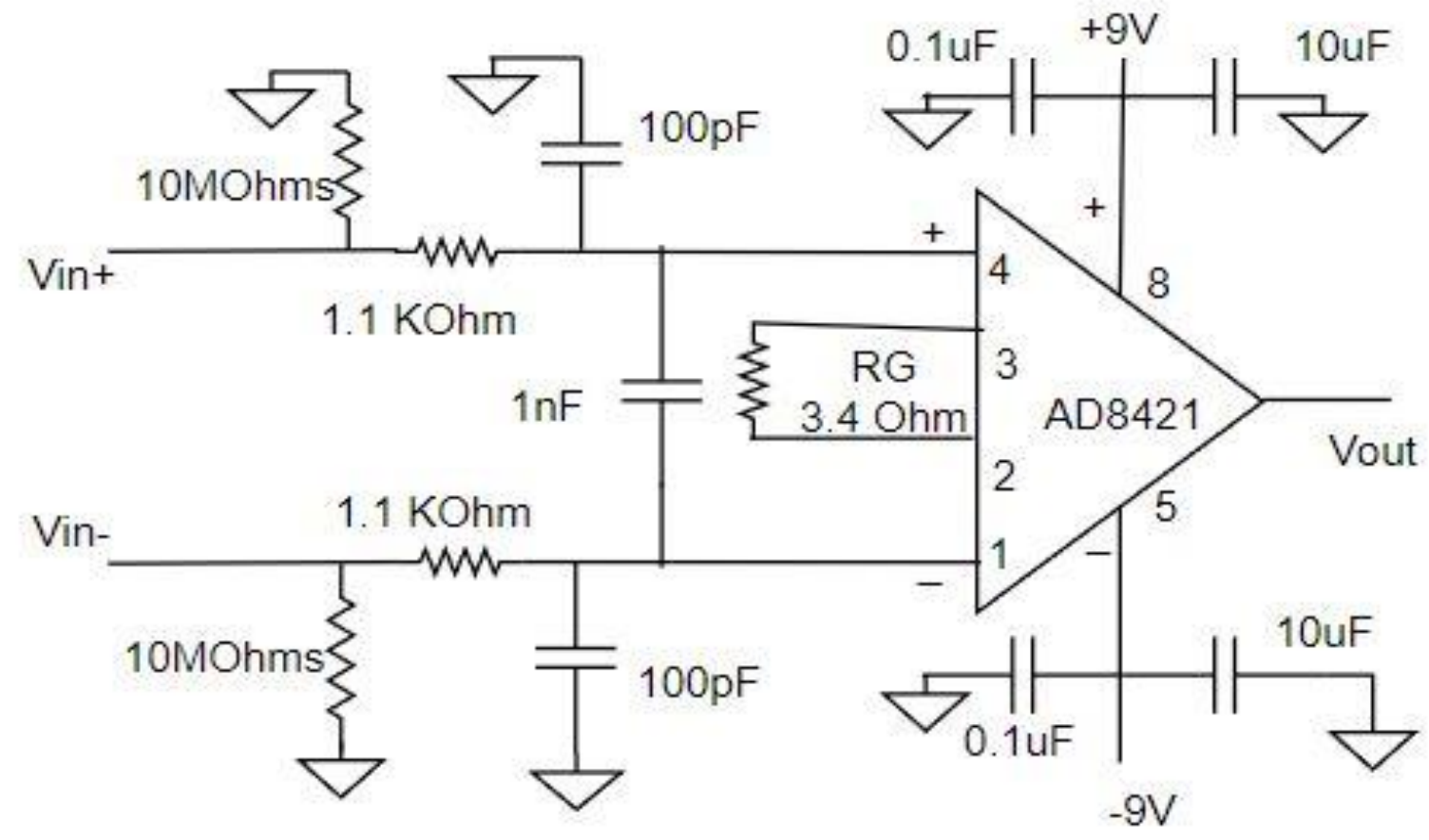

Figure 12, Low noise amplifier circuit with 2912 gain

AN-1264 has a series resistor between input and AD8421, this resistor protects the input from over voltage and radio frequency interference. In this project $4.7 \mathrm{KOhm}$ resistor is replaced with $1.1 \mathrm{KOhm}$ resistor to reduce the noise and resistance for very low voltages. Total noise for the input resistance is

$$
\sqrt{2(4 * \sqrt{1.1})}=5.93 \mathrm{nV} / \sqrt{\mathrm{Hz}}
$$

Voltage noise of amplifier is calculated as

Total Voltage Noise $=\sqrt{\left(\frac{\text { outputNoise }}{G}\right)^{2}+(\text { InputNoise })^{2}+(\text { Noise of } R G)^{\wedge} 2}$ 
Total Voltage Noise $=\sqrt{\left(\frac{60}{100}\right)^{2}+(1.7)^{2}+(4 * \sqrt{(0.0034)})^{2}=2.87 \mathrm{nV} / \sqrt{\mathrm{Hz}}}$

Current Noise of the amplifier $=\sqrt{\left((1.1 * 0.2)^{2}+(1.1 * 0.2)^{2}\right)}=0.31 \mathrm{nV} / \sqrt{\mathrm{Hz}}$

Total Noise Density $=\sqrt{\left(5.93^{2}+2.873^{2}+0.31^{2}\right)}=6.596 \mathrm{nV} / \sqrt{\mathrm{Hz}}$

\subsection{Filters:}

\subsubsection{Introduction:}

A filter is a two-port electrical circuit that, according to its design, attenuates frequency components from a signal presented to its input port. Filters are classified according to their function, component composition, linearity, and impulse response, among other characteristics. Several of these are discussed below.

Active and Passive filters:

Active filters are electrical circuits that utilize active components such as amplifiers. Amplifiers may significantly increase a filter's cost, performance, and predictability. A passive filter is an electrical device constructed entirely of passive components such as resistors, capacitors, transformers, and inductors [39].

Analog and Digital filters:

Analog filters are constructed using analog components such as resistors, capacitors, inductors, and operational amplifiers (op amps). Digital devices are frequently included into digital signal processing chips, such as an Microcontroller Unit, System on a chip, Central Processing Unit [44].

Low pass, high pass, band pass, and band stop filters are all examples of analog filters. An active low pass filter is employed in this project to reject frequencies greater than $10 \mathrm{~Hz}$.

Low Pass filter: Low pass filter is an electronic device which only accepts the frequencies below the cut-off frequency and attenuates the higher frequencies.

High Pass filter: A high pass filter allows frequencies higher than a certain frequency and attenuates frequencies below a certain frequency. 
Band Pass filter: A band pass filter accepts frequencies within a band and attenuates frequencies above and below that band.

Band stop filter: Band stop filter attenuates frequencies within that band, allows frequencies above and below that band.

An active low pass filter is utilized in this project to efficiently reduce $60 \mathrm{~Hz}$ noise. A typical low pass filter is composed of passive components such as capacitor and resistor.

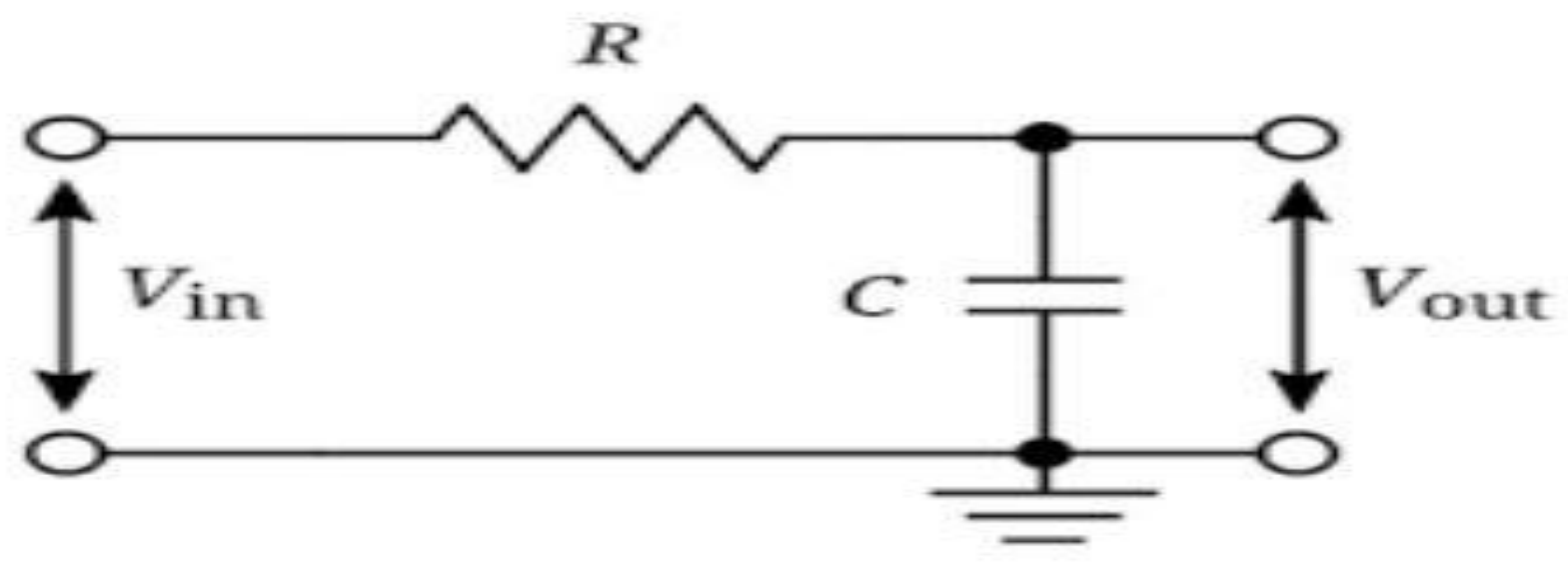

Figure 13, passive low pass filter[45]

Because these passive components lack amplifiers, they provide no signal gain. Gain is always less than or equal to unity in this case, and the output level is less than the input level. In this case, the input is applied across both the resistor and the capacitor, while the output is taken across only the capacitor. This sort of filter is referred to as a first order filter or one-pole filter since it has only one reactive component, the capacitor. The reactance of a capacitor fluctuates inversely with frequency, whereas the resistor's value remains constant. At low frequencies, the capacitor's capacitive reactance (Xc) will be extremely big in comparison to the resistive value of the resistor, $R$. As a result, the voltage potential across the capacitor is significantly greater than the voltage drop across the resistor. At high frequencies, voltage drop across capacitor becomes tiny and voltage drop across resistor becomes big as the capacitive reactance value changes [44].

The primary drawback of passive filters is that the gain is always less than unity and that the filter properties are affected by the load impedance. With multiple-stage passive filter circuits, this loss in signal amplitude, referred to as "Attenuation," can become fairly severe. One technique to restore or limit this signal loss is to use an active filter that has by definition, amplification. 
Active filters incorporate operational amplifiers and field effect transistors into their circuit architecture. They are powered externally and are used to boost or amplify the output signal. Additionally, filter amplification may be used to shape or adjust the frequency response of the filter circuit by providing a more selective output response, so narrowing or widening the filter's output bandwidth. The primary distinction between a "passive filter" and an "active filter" is therefore one of amplification.

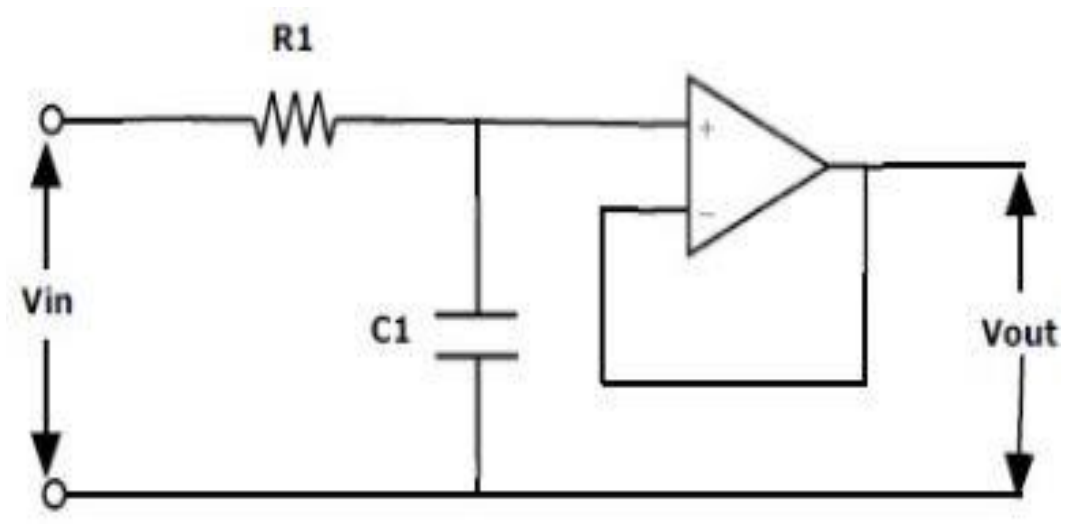

Figure 14, Active Low Pass Filter [46]

The first order active low pass filter is basically a passive $\mathrm{RC}$ filter stage that connects the input of a non-inverting operational amplifier to the low frequency channel. Here, the low output impedance of the op amp prevents the filter's cut-off frequency point from being impacted by changes in the load's impedance. The voltage gain is one in this setup, but the output is unaffected by load impedance. We may use the following circuit to increase the voltage and gain.

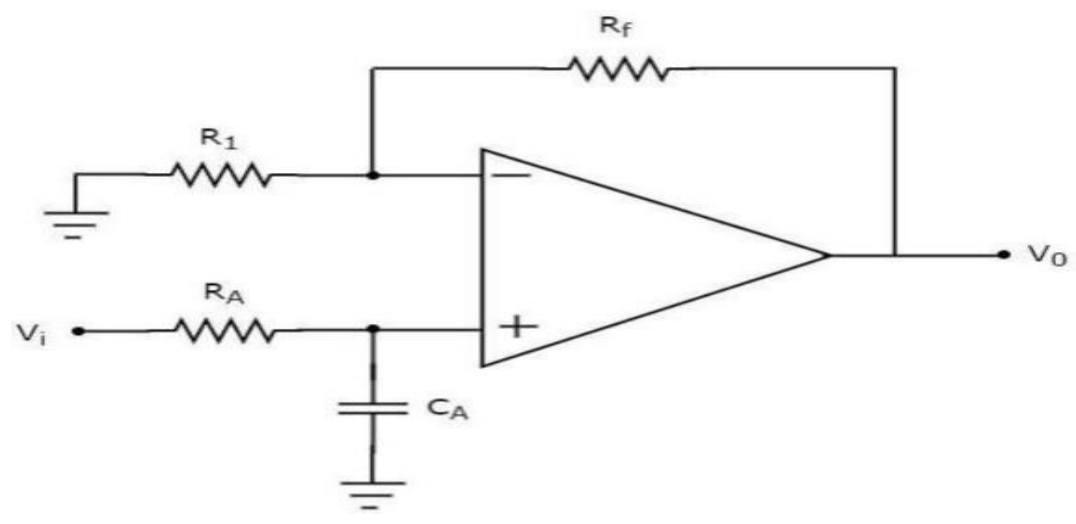

Figure 15, Active low pass filter with amplification [47]

The gain of the circuit response is identical to that of an $R C$ circuit, but the amplifier's voltage gain is boosted by a factor of $\mathrm{Rf} / \mathrm{R} 1$ [44]. 


\subsubsection{Second order filters:}

Second order filters, also known as Voltage Controlled Voltage Source (VCVS) amplifiers, are another form of active filter that is widely used. Converting first order filters to second order filters is as simple as adding an additional RC network to the input or feedback channel. Second order filters may be defined as two first order filters cascaded with amplification.

The Sallen-Key filter is a well-known and common second order filter design that consists of a single operational amplifier and four RC components. The Sallen-Key filter with unity gain is illustrated below.

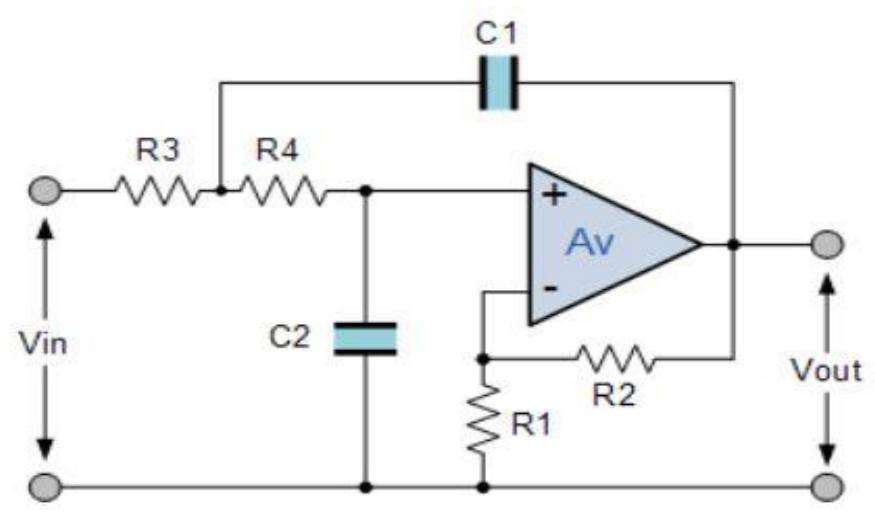

Figure 16, Sallen-Key Low Pass filter [49]

The $Q$ factor, which specifies the height and width of the filter's frequency response, is equal to $\sqrt{\frac{(R 3 * R 4 * C 1 * C 2)}{C 2 *(R 3+R 4)}}[52]$. The filter's cutoff frequency is calculated using the formula $\frac{1}{2 * \pi * \sqrt{R 3 * C 1 * R 4 * C 2}}[47]$.

Gain at the filter circuit can be obtained using the feedback resistors. Formula for gain to second order low pass filter is Gain $=1+R 2 / R 1$ [47].

\subsection{Analog Devices - AD8510:}

\subsubsection{Introduction:}

The AD8510 is a single precision JFET amplifier with a low offset voltage, low input bias current, low input voltage noise, and low input current noise. Due to their low offsets, low noise, and extremely low input bias currents, these amplifiers are particularly well suited for amplification of high impedance sensors and precise current measurements employing shunts. When combined with minimal noise and a short settling time, dc precision leads in improved accuracy in medical devices, electronic measurement, and automated test equipment. Even with large capacitive loads, these 
amplifiers retain rapid settling performance. "The AD8510's rapid slew rate and excellent stability with capacitive loads make it an ideal candidate for high performance filters. The AD8510 is a good choice for audio applications due to its low noise and distortion, high output current, and fast speed. The AD8510's low input current noise and input bias current make them appropriate for circuits with a high input source resistance. At normal temperature, the input offset voltage rises by less than $15 \mathrm{nV}$ for every 500 ohms of source resistance.

Settling time is the time required for the amplifier's output to attain and maintain a percentage of its ultimate value following the application of a pulse at the input. With a step of $0 \mathrm{~V}$ to $10 \mathrm{~V}$ in unity gain, it settles in less than $900 \mathrm{~ns}$. As a result, AD8421 is a good candidate for serving as a buffer at the output of DACs with a typical settling time of less than 1us" [48].

"Overload recovery time is the time required for an amplifier's output to return to its linear region following a saturated situation. This recovery time is critical in situations where the amplifier must enhance low-level signals in the presence of high-voltage transients. From a saturated state, the output recovers in around $200 \mathrm{~ns}$. It has exceptional symmetry in terms of both positive and negative recovery durations. This is a critical characteristic for transient signal rectification since it ensures that the output signal remains undistorted for any given duration. Even when driving large resistive and capacitive loads, it has a great loop gain and phase response" [48].

Pin diagram: Figure 17 shows pin diagram of AD8510.

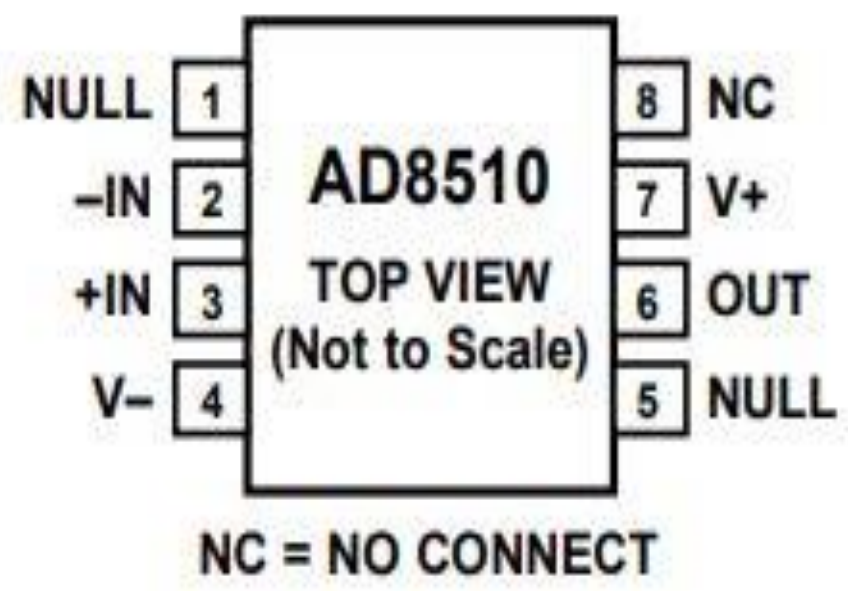

Figure 17, AD8510 pin diagram[48] 


\subsubsection{Example Filter circuit in Analog Devices (AN-1264) specification sheet:}

The low pass filter employed in the AN-1264 using analog devices is shown below; it is a second order Sallen-Key filter. The AD8510 is used for filtering, as it is a low noise JFET operational amplifier with an $8 \mathrm{nV} / \mathrm{Hz}$ voltage noise density. It has a $460 \mathrm{~Hz}$ corner frequency. Due to the voltage divider effect of the 20KOhm resistors, the filter's output will be 0.5 times the input voltage. At low frequencies, capacitors are open; at higher frequencies, capacitors are shorted, thereby removing higher frequencies [43]. Circuit shown in Figure 18 gets input from amplifier circuit and output goes to an Analog to Digital converter.

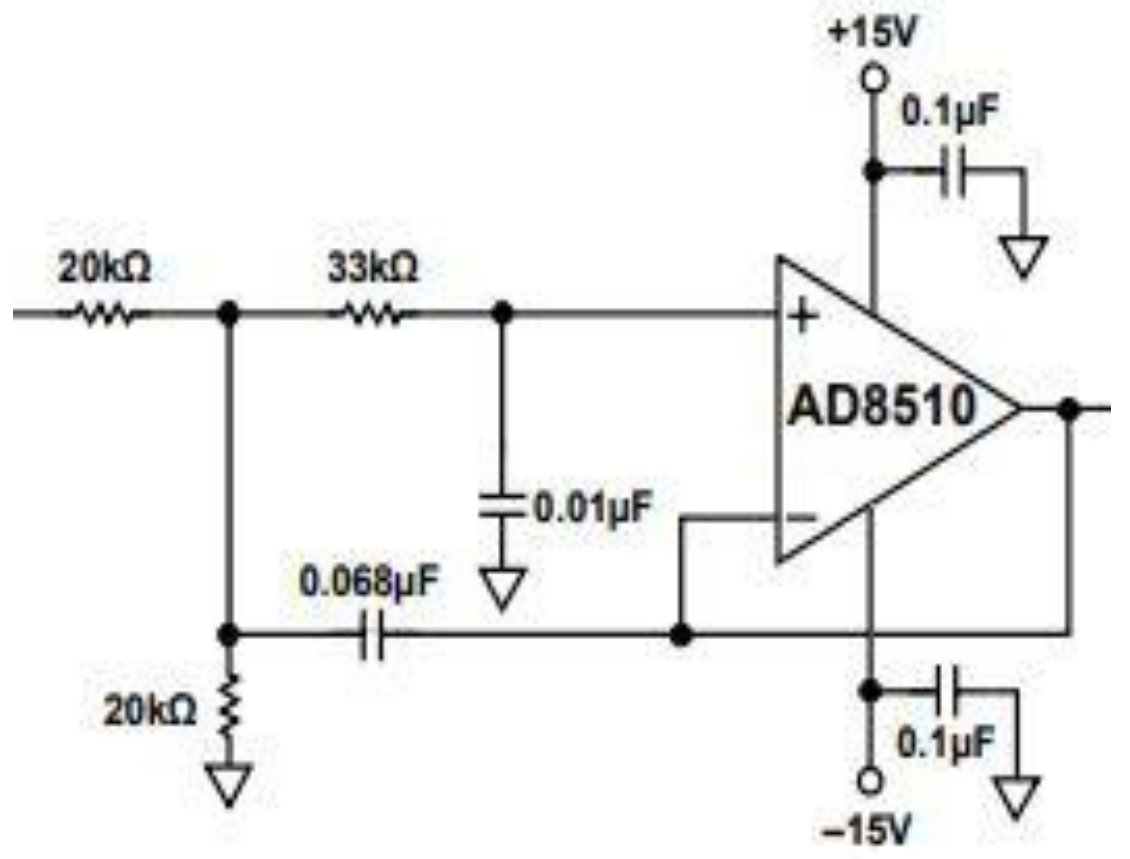

Figure 18, AN-1264, Low Pass Filter [43]

\subsubsection{Redesign of filter:}

Resistors and capacitor values have been chosen to match the specified cut-off frequency and damping factor. To generate a clean output wave, a cut-off frequency of $10 \mathrm{~Hz}$ and a damping factor of 1 are utilized in this circuit. The voltage divider circuit has been eliminated to prevent the output voltage from falling to 0.5 times the input voltage. 


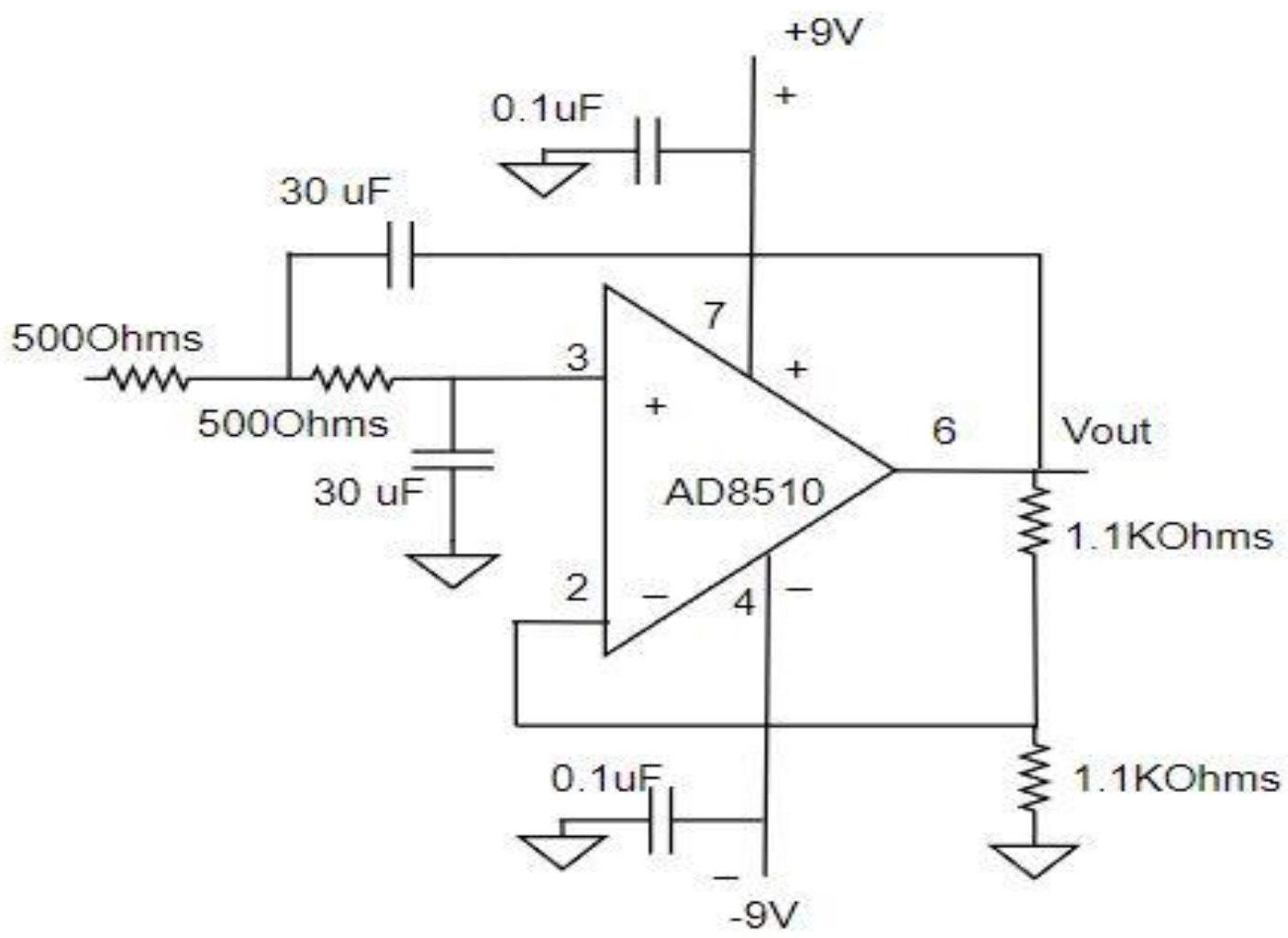

Figure 19, Low pass filter with $10.6 \mathrm{~Hz}$ cutoff frequency

Quality factor $Q$ is set to 1 in this project and thus a gain of 2 is obtained. To obtain a gain of 2, resistors $1.1 \mathrm{KOhms}$ are connected at the feedback loop.

Gain $=1+(1.1 \mathrm{KOhm} / 1.1 \mathrm{KOhm})=2$

Cut-off frequency fc $=\frac{1}{2 * \pi * R * C}=\frac{1}{2 * \pi * 500 * 30 *\left(10^{6}\right)}=10.6 \mathrm{~Hz}$

This filter only reduces the amplitude of noise, but it doesn't eliminate the noise totally. A second low pass filter is connected in series to the output of the first low pass filter which attenuates the electrical noise significantly giving a sine wave with lesser noise amplitude.

\subsection{Overall circuit:}

The overall circuit for this design is shown in Figure 20. This design has an amplifier and two low pass filters connected in series. The amplifier amplifies the signal with a gain of 2912 and the filters attenuate the electrical noise. At every filter stage, the filter amplifier has a gain of 2 . 


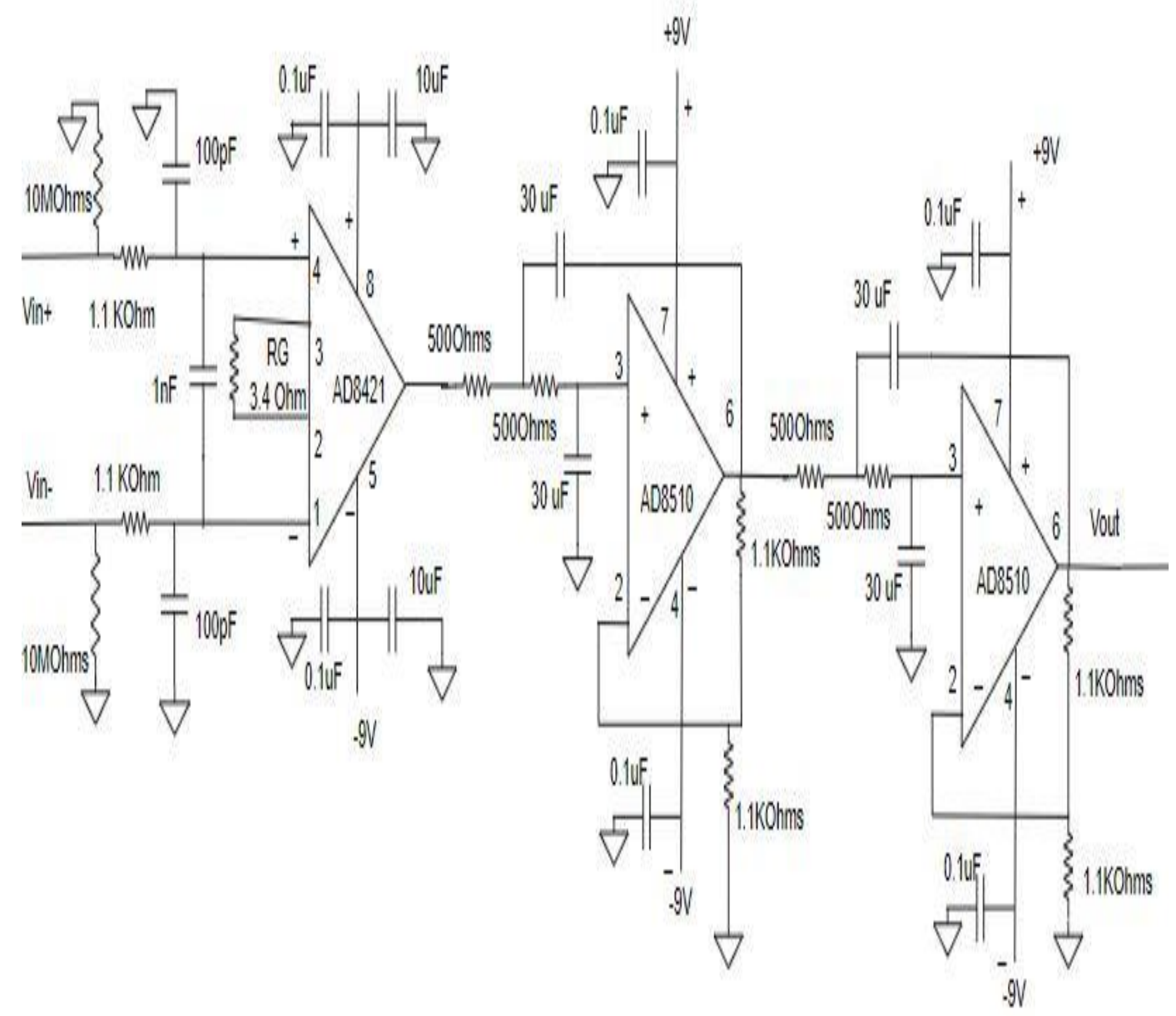

Figure 20, Redesigned circuit diagram 
A Signal generator is used to generate sine wave at $7 \mathrm{~Hz}$ frequency, output of signal generator is connected to small coil which generates sine waves and induce those waves to the Inductor-Capacitor coil connected at the input of amplifier as shown in Figure 21. Lowest voltage that signal generator can supply is $10 \mathrm{mV}$. Due to the inductance between the smaller coil and LC circuit, input voltage before amplifier is in 100 's of micro volts. This input voltage was brought down further by moving the smaller coil away from the LC circuit.

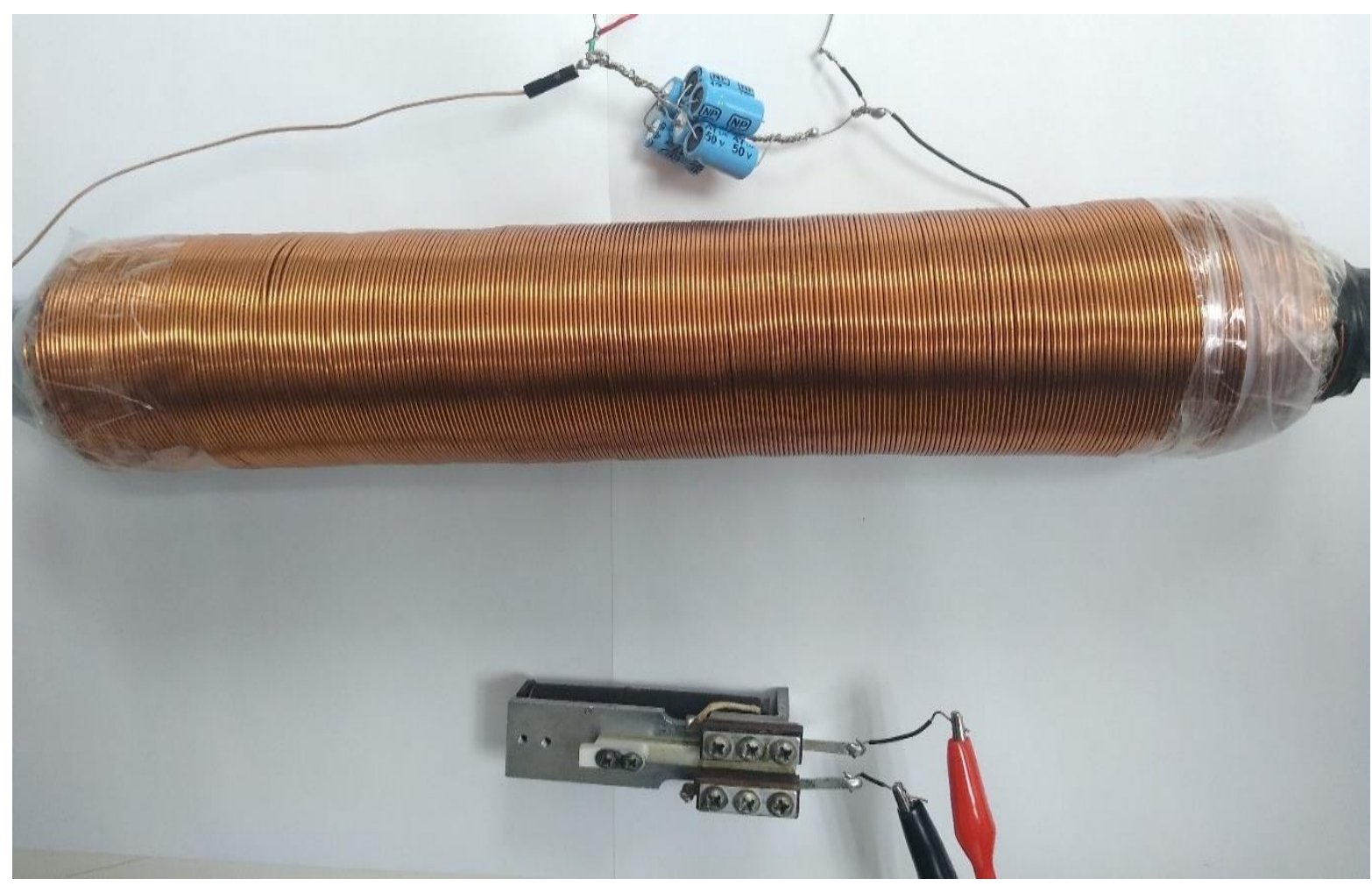

Figure 21, smaller coil induces signals to larger coil(LC circuit)

Figure 22, shows the overall circuit of how signal is fed to amplifier and output is taken out of filter using oscilloscope. 


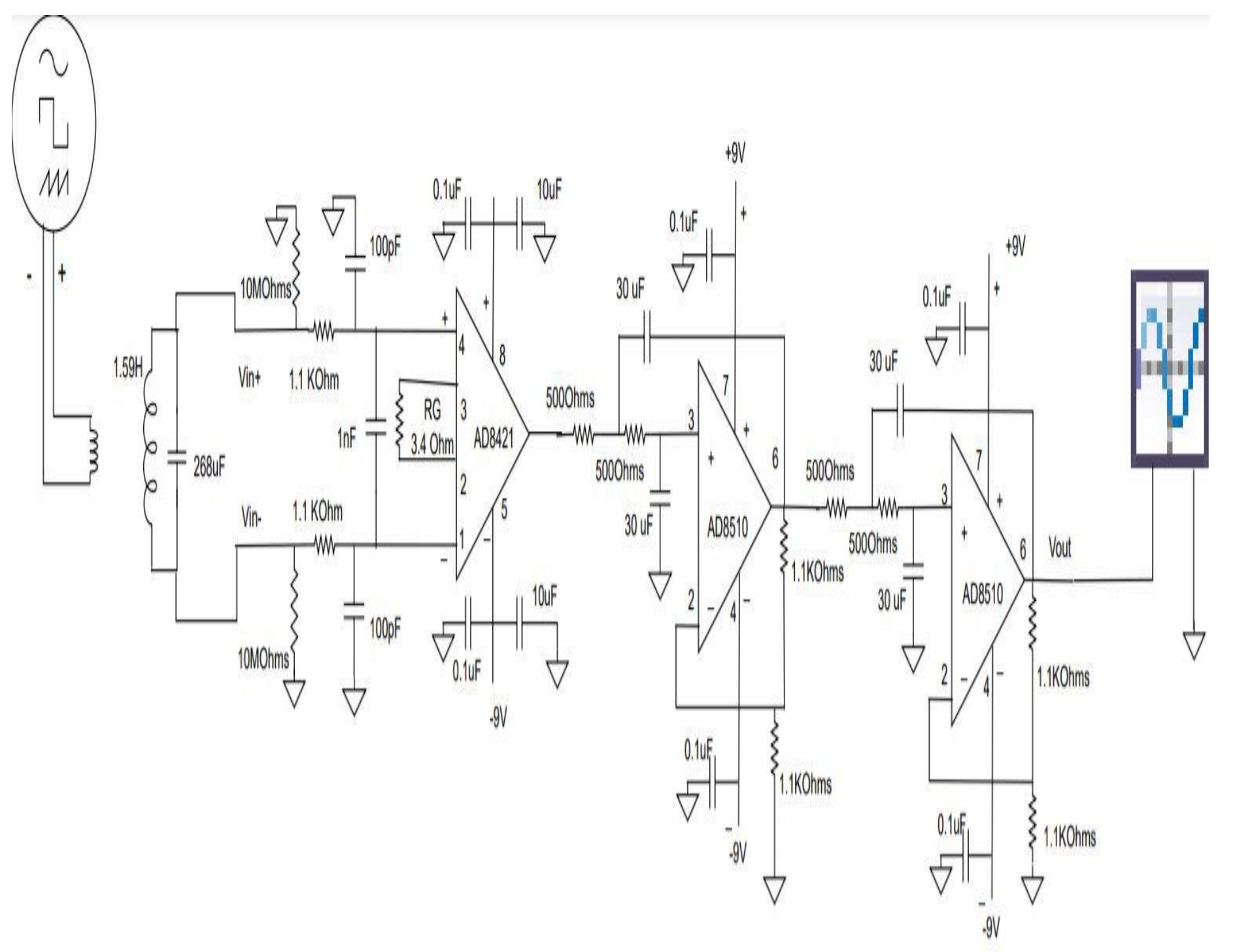

Figure 22, overall circuit of the project

\section{Chapter 3 \\ PC Board:}

\subsection{Schematic Design:}

The circuit was implemented on a breadboard following the initial design. After finishing the circuit, a printed circuit board was designed to minimize wiring. At $7 \mathrm{~Hz}$, lengthy cables and component leads can produce significant distractions in the output signal as a result of contact with electrical noise and frequencies in the atmosphere with high signal strength. Eagle CAD PCB design software was used to create the design's schematic circuit and printed circuit board layout. The design is schematically depicted in Figures 23, 24. Total Schematic design has been divided into two part due to size 
limitations of the document. Figure 23 shows Amplifying stage and Figure 24 shows Filtering stage.

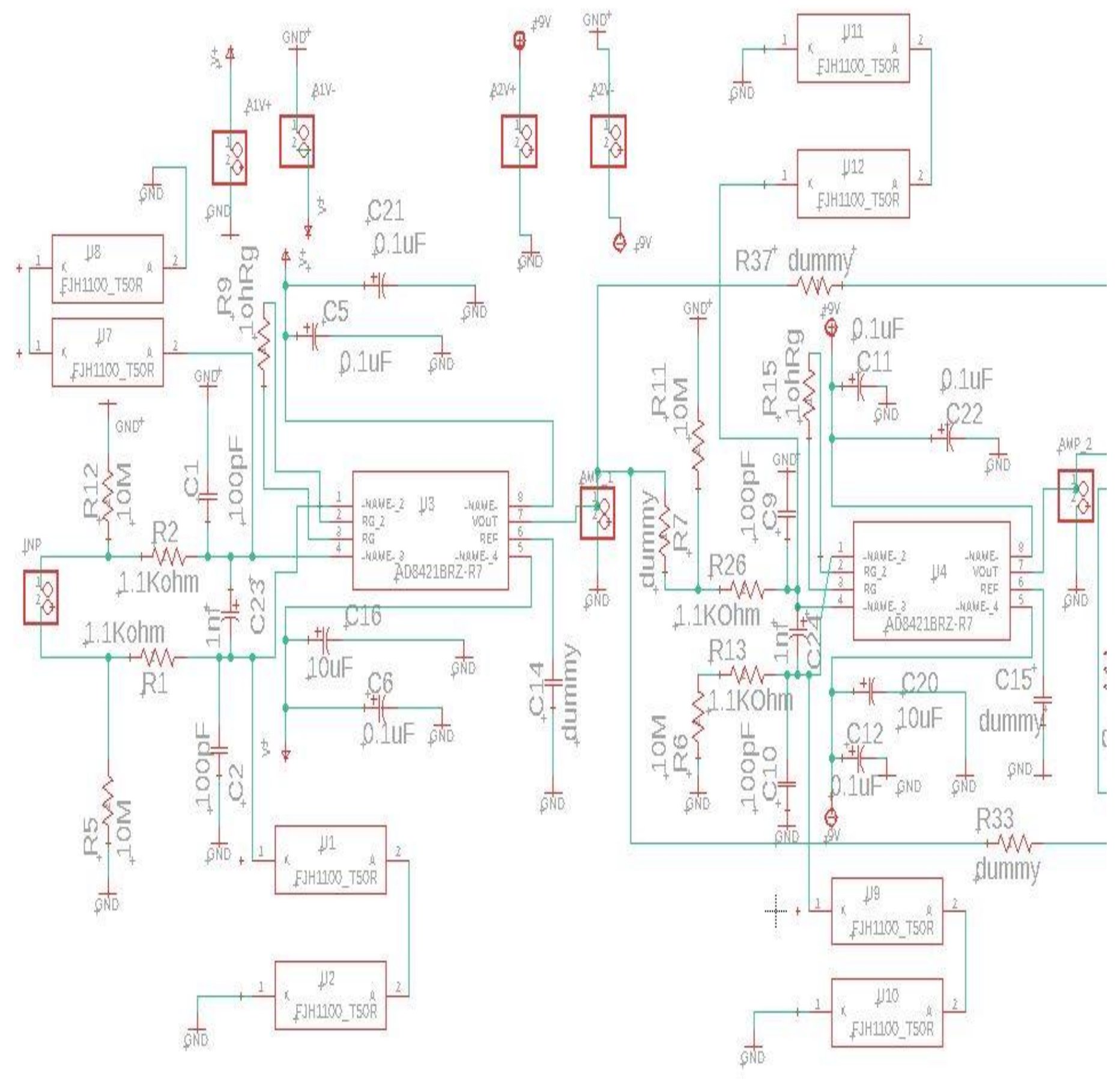

Figure 23, Schematic design of the Amplifiers Stage 


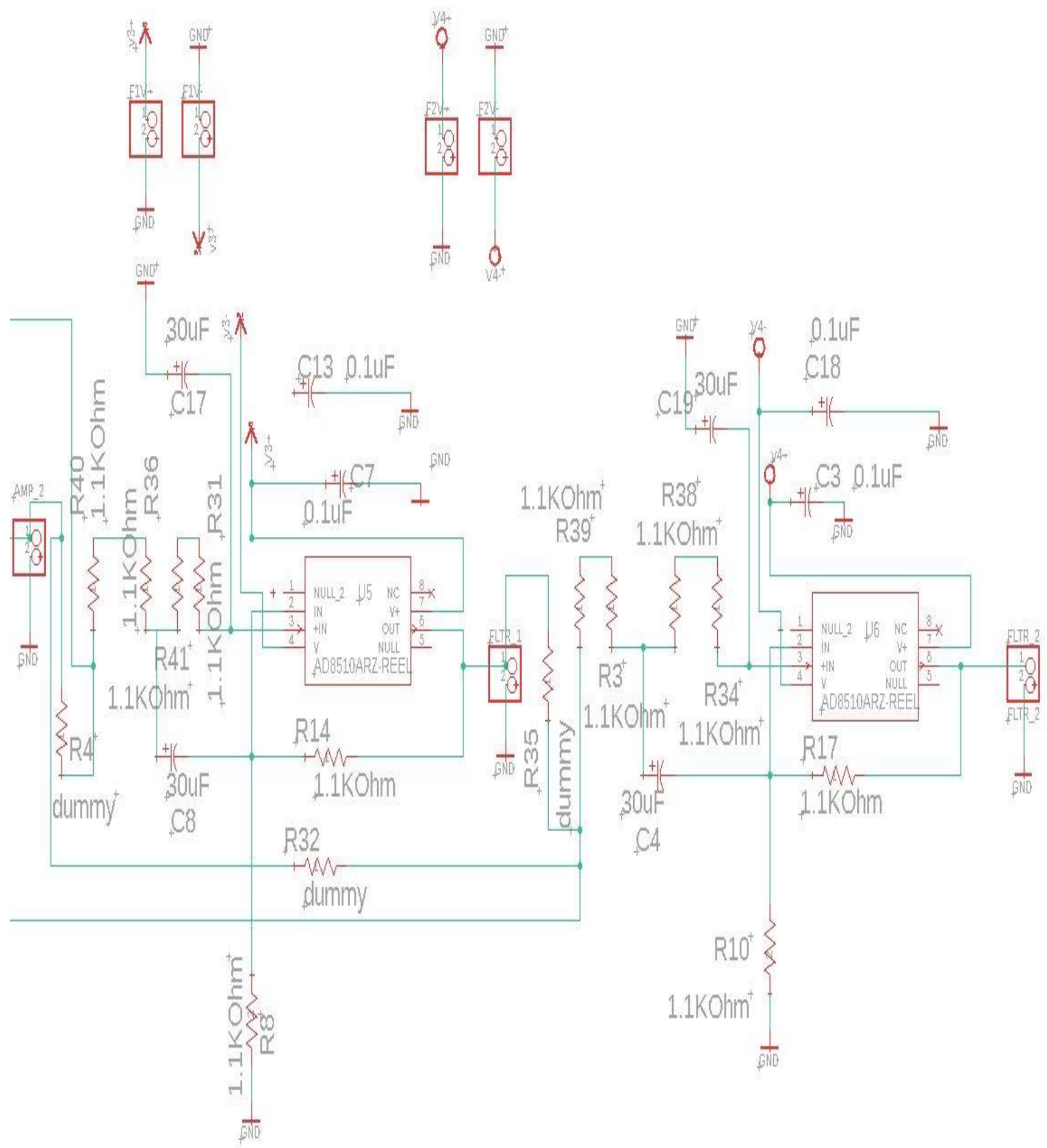

Figure 24, Schematic Design of the circuit Filters stage 


\subsection{Board Design:}

This Schematic diagram is transformed into a printed circuit board layout using AutoCAD Eagle. The process is as follows. Initially on the computer screen, all components are placed outside the actual board area. Components are placed one after another one by one on the board layout. One goal of the layout is to place components in such a way that they occupy the minimal amount of board space and then so that the copper circuits or "Lan" routing between chips is easy for the designer. Once all the components are placed on the board, then "air wires" are routed between chips. The routing wires cannot overlap one another so that if possible, all routing is on one layer of the pc board. The board design chosen here is a double layer design. One side of the board is completely allocated to the ground conductor in order to help shield and isolate the sensitive amplifiers from noise. Having a solid ground reduces internal electrical noise coupling and reduces interference from ground loops and cross talk between adjacent circuits. All of this is called Electro Magnetic Interference of EMI. The board design is shown in Figure 24.

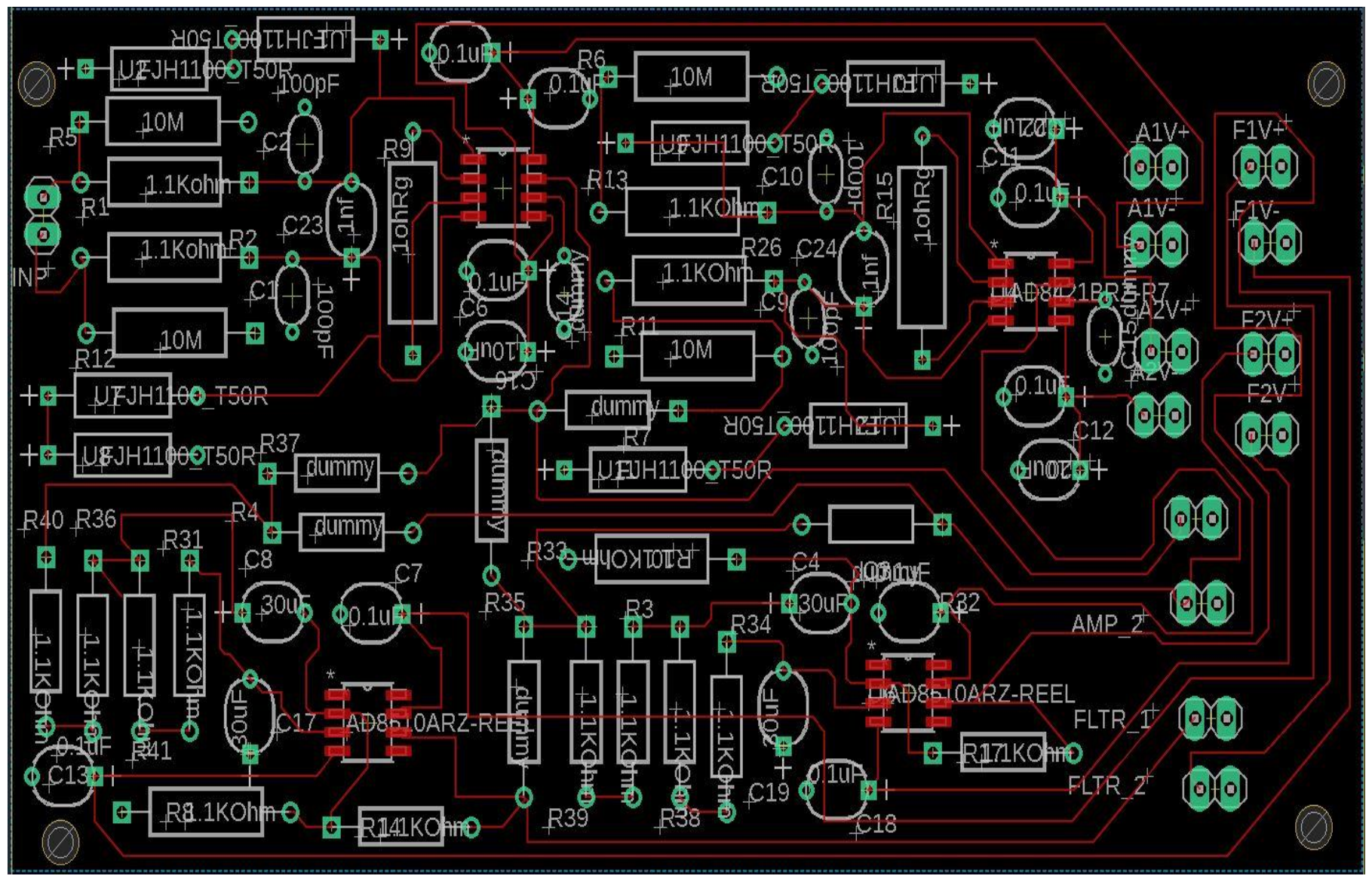

Figure 25, PCB design before manufacturing 
3.2 PC board:

This layout design was translated to a printed circuit board layout. Following that, the PCB design files were translated to "Gerber format." These files were then used to cut out the real board by using the ProtoMat and PromatLaser machines located in the WVU innovation Lab. The WVU iLab offers instruction on how to design a PCB using ProMat equipment. This training session lasts around four hours and includes a drilling phase, a masking phase, a plating phase, and a trace marking phase. The holes were drilled using a ProMatSL machine. Masking and plating were done manually; trace marking was carried out with the aid of a ProMatL machine. Figure 25 shows the manufactured PC Board.

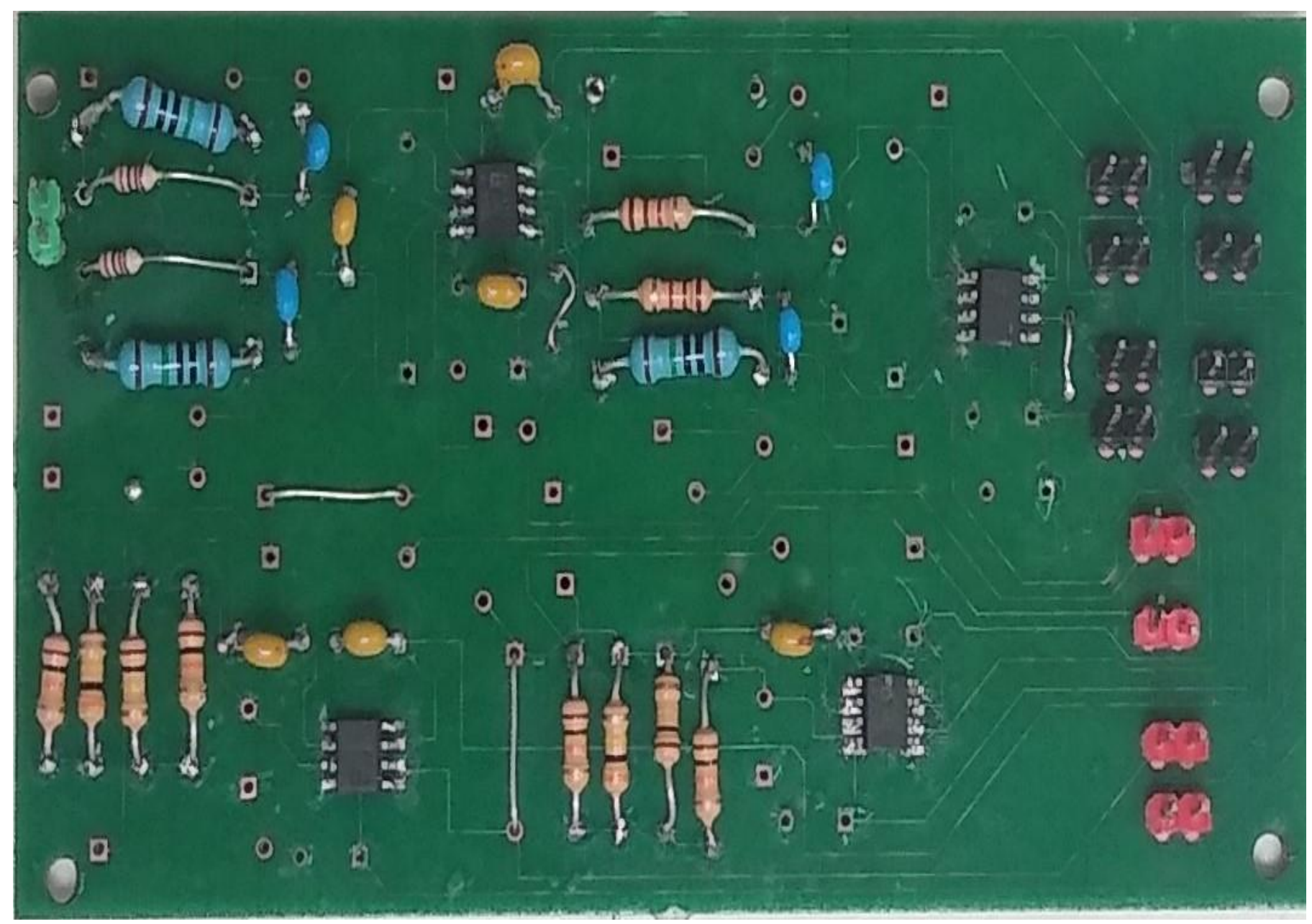

Figure 26, Printed Circuit Board

The PCB was constructed as a double-sided board, with the bottom side entirely dedicated to the ground conductor. By dedicating a single side to the ground, the PC board becomes less reactive to atmospheric signals, as the ground terminal reduces the higher frequency impedance thus attenuating noise. 


\section{Chapter 4 \\ Design characteristics:}

\subsection{Output waveforms}

\subsubsection{Amplifier Stage:}

The amplifier is employed in the first stage to boost the strength of the signal from the LC tuned circuit. The LC circuit's output voltage (which also works as an antenna) is between $2 \mathrm{uV}$ and $400 \mathrm{uV}$ and fed to the initial amplifier on the PCB. Due to their modest strengths, these signals can be very noisy. The amplifier was used to boost signal intensity and then send its output through the filters to reduce electrical noise. The following illustration in Figure 26 depicts the output waveform following the amplifier.

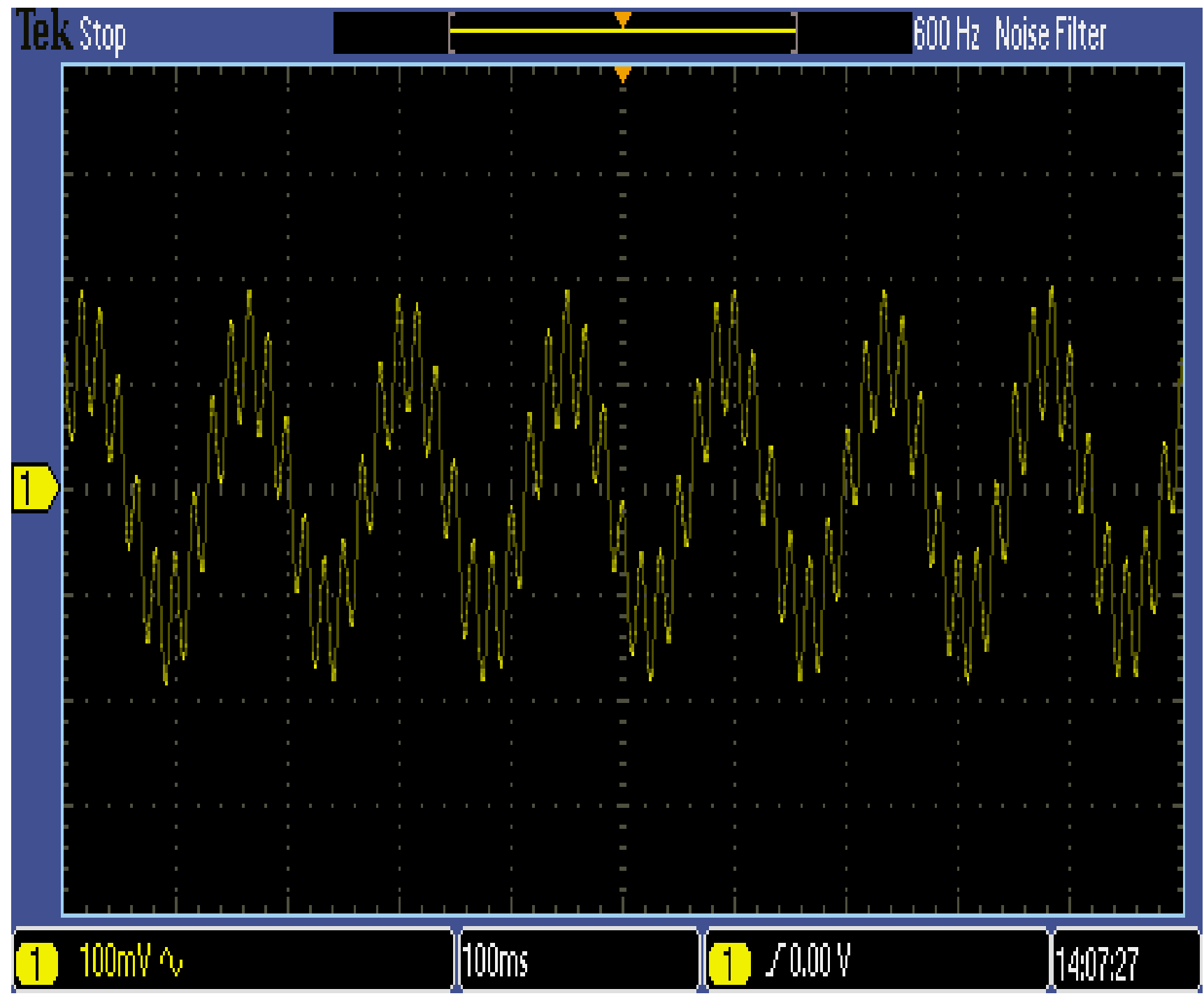

Figure 27, Output after amplifier circuit 


\subsubsection{First stage Low Pass filter stage}

With a low amplitude signal from the LC and amplifier, connecting a filter to the output of the amplifier reduces the amplitude of electric noise. Output after the stage 1 low pass filter is shown in Figure 27.

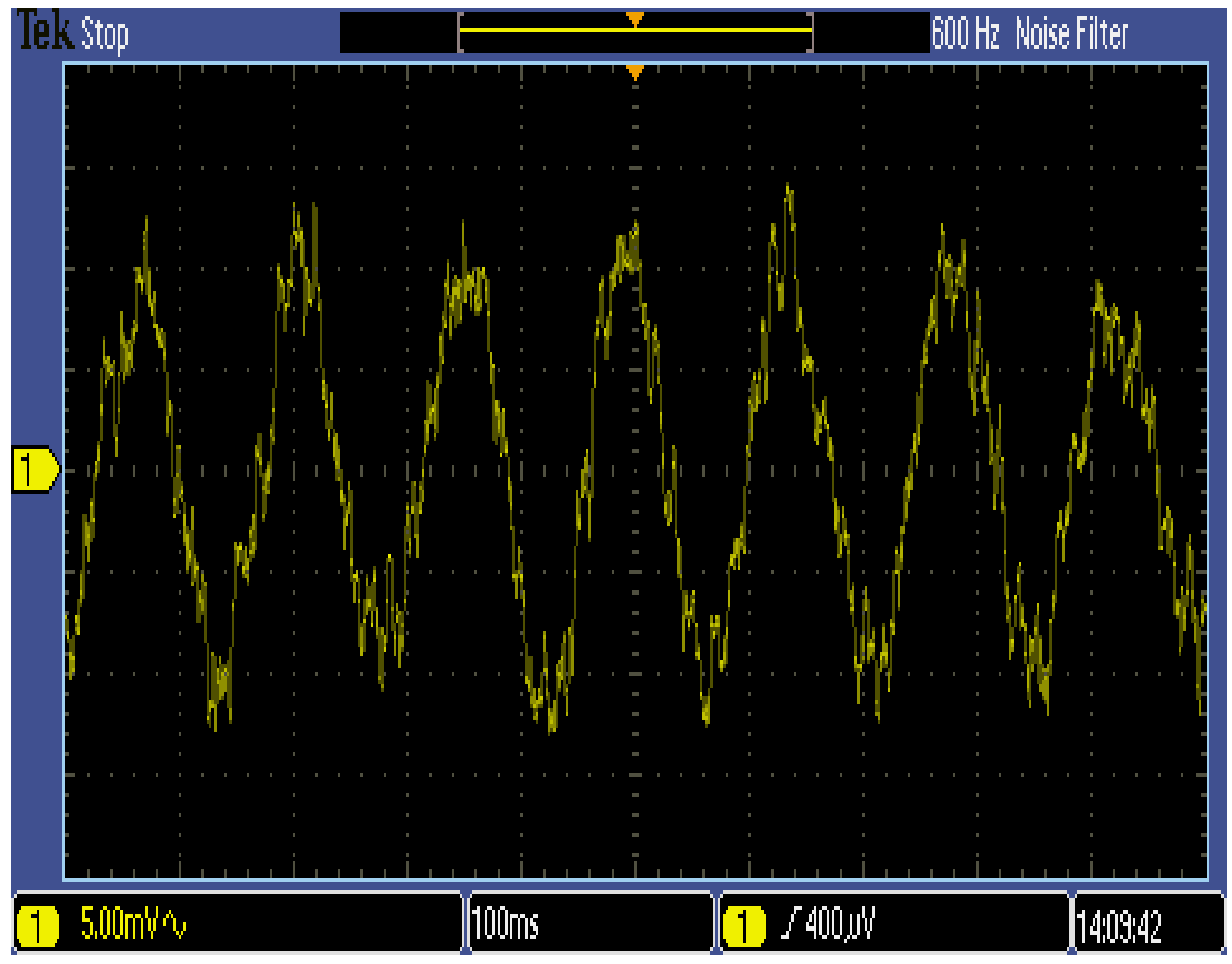

Figure 28, Output after low pass filter stage 1

\subsubsection{Second stage Low Pass filter stage}

Connecting the second low pass filter at the output of the first low pass filter gives a clear sign wave as shown in Figure 24. 


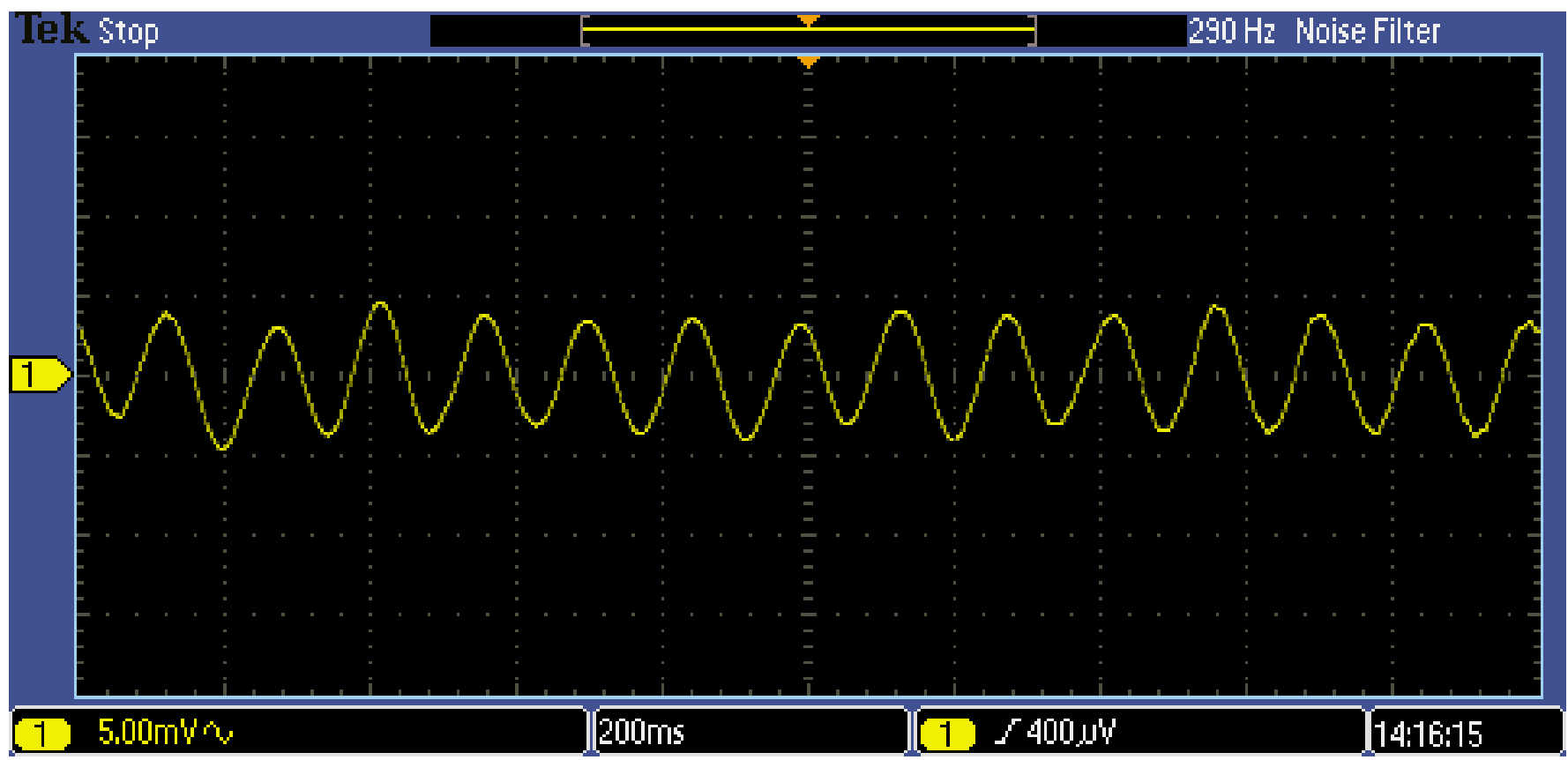

Figure 29, Output after filter stage 2

\subsection{Received Data:}

Voltage vs frequency and input vs output voltage characteristics are plotted in Figure 26. Input voltage is obtained using oscilloscope at the end of LC circuit. Due to the limitations of the work equipment, input voltage are read clearly only until a few uV. Input voltages below are reverse calculated using gain and output voltage.

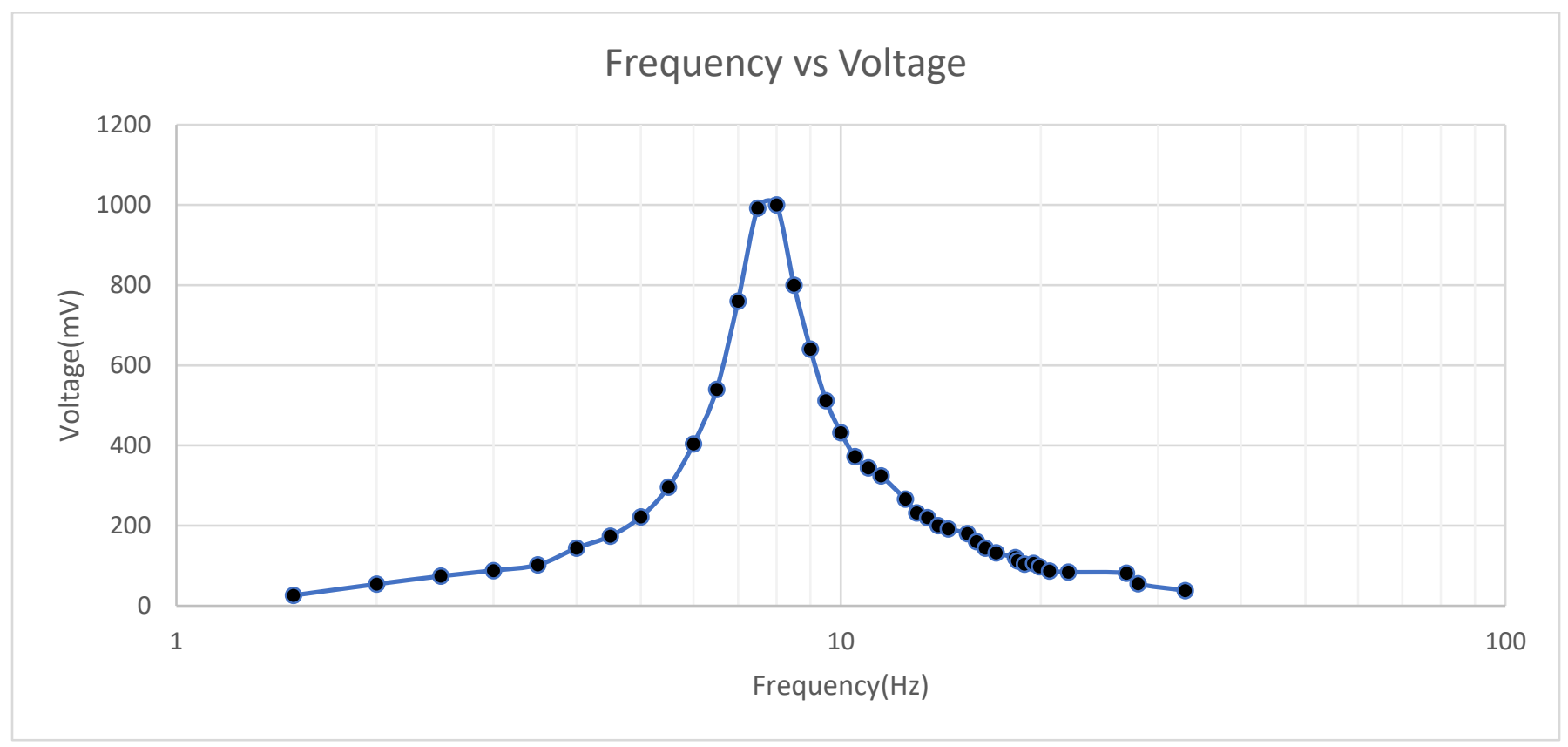

Figure 30 , voltage vs frequency graph 


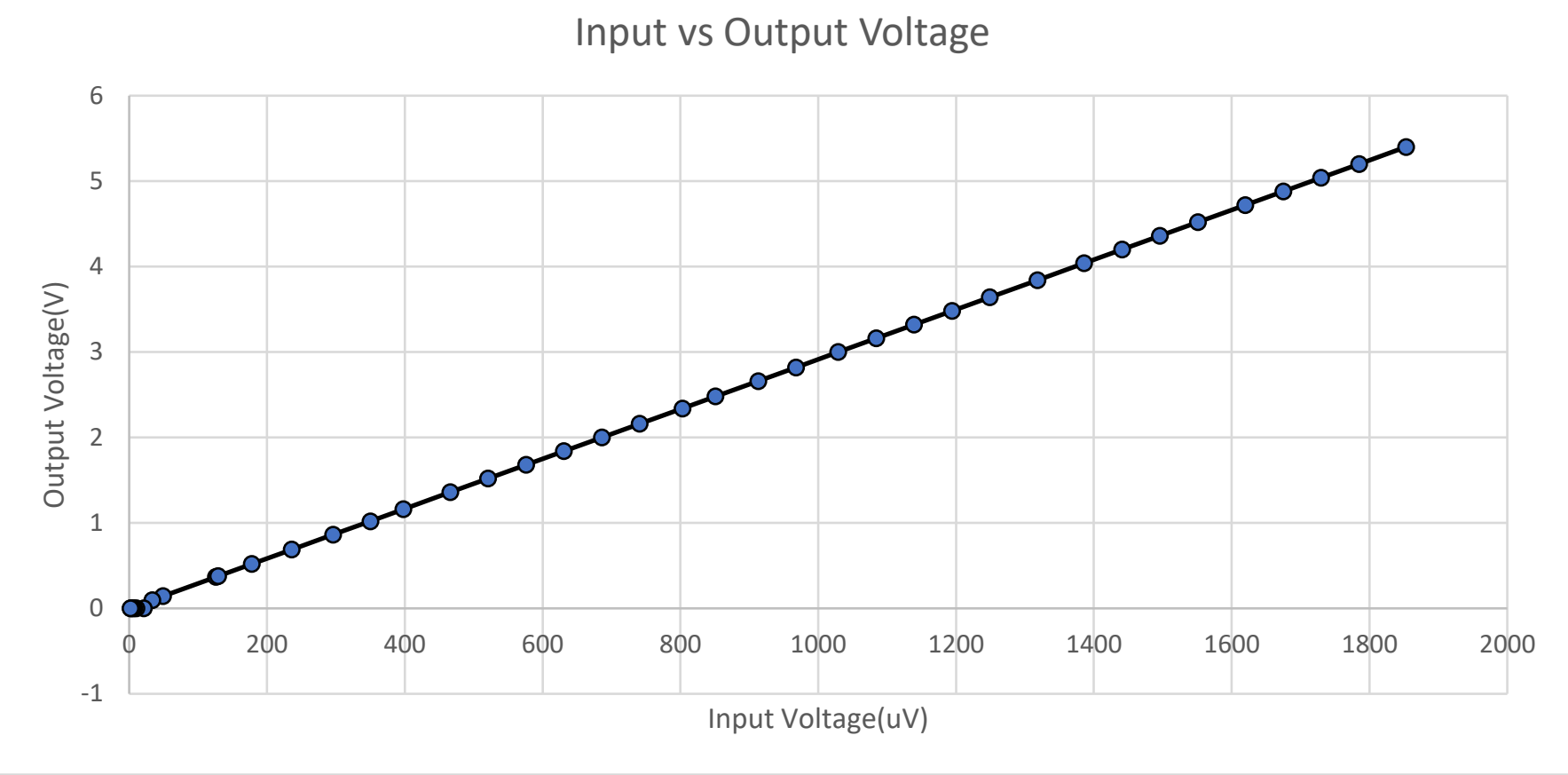

Figure 31, Input voltage vs Output voltage

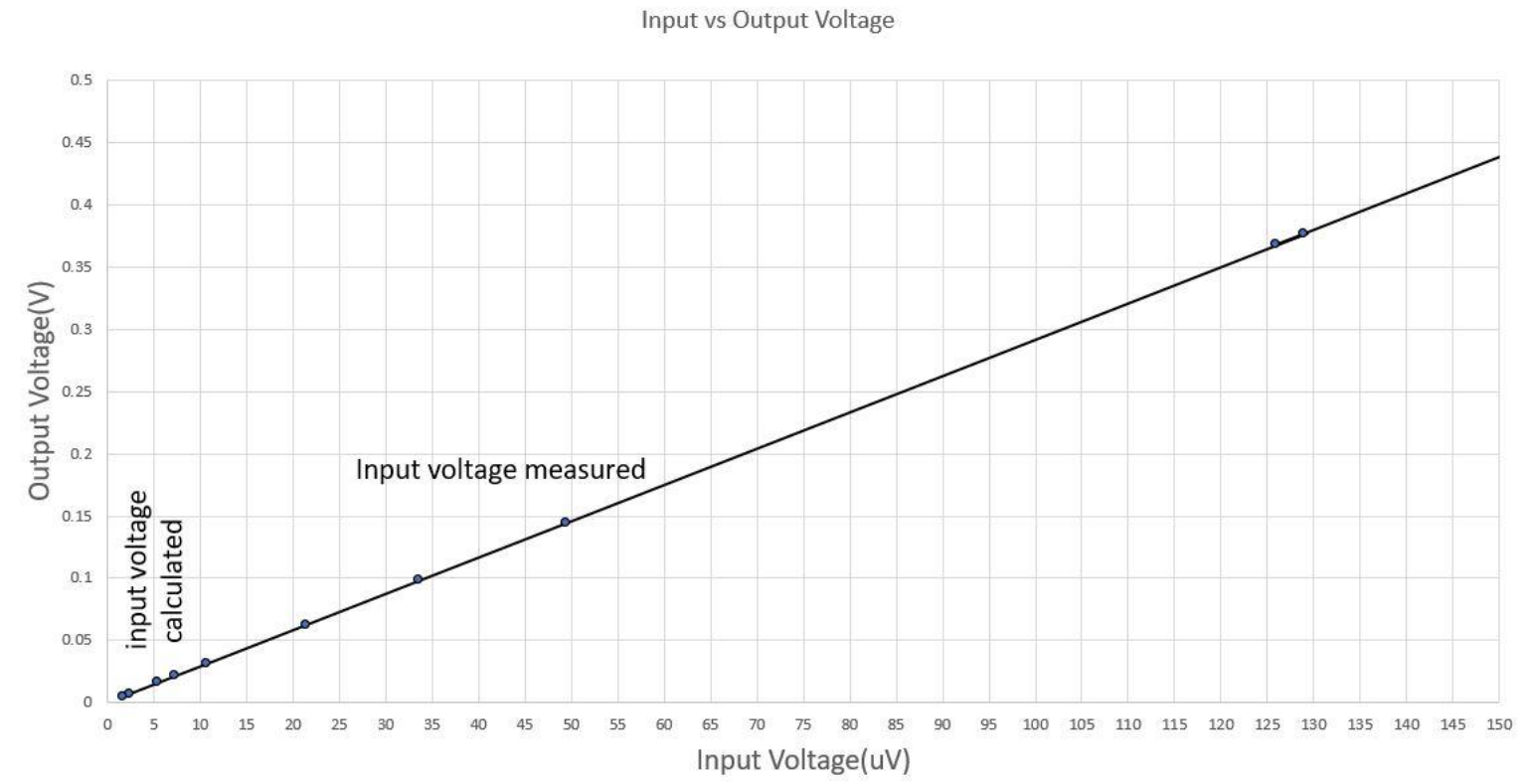

Figure 32, Input voltage range $(1.73 \mathrm{uV}-350 \mathrm{VV})$ vs Output voltage range (5mV$1.02 \mathrm{~V})$ 


\subsection{Conclusion:}

Redesigned the ancillary circuit surrounding the Analog Devices AN-1264 with suitable voltage gain for Schumann Resonance frequencies including the amplifier, cutoff frequency and damping factor for the low pass filter. Efficiently detected and amplified signals around $2 \mathrm{uV}$, eliminated $60 \mathrm{~Hz}$ electrical noise obtaining a clear sine wave.

\section{Chapter 5}

\subsection{Future work:}

Practical implementation of this circuit in the field packaging to try to detect the Schumann Resonance signals.

\subsection{Bibliography:}

1. NASA. "What are the spectrum band designators and bandwidths", [08-10-2021]. [Accessed on: 11-30-2021]. Available: https://www.nasa.gov/directorates/heo/scan/communications/outreach/funfacts/tx t_band_designators.html.

2. Price, Colin.. "ELF Electromagnetic Waves from Lightning: The Schumann Resonances" 2017, Atmosphere 7, no. 9: 116.

3. Barr, R.; Stubbe, P. ELF and VLF radiation from the "polar electrojet antenna". Radio Sci. 1984, 19, 1111-1122.

4. M. Feychting, and A. Ahlbom, "Magnetic fields and cancer in children residing near Swedish high-voltage power lines," Am. J. Epidemiol., 1993, Vol. 138, pp. 467-481.

5. Ronald W. P. King., Electromagnetic Fields in Human Body Due To VLF Transmitter, Antennas and Propagation Society International Symposium, 1996.AP-S. Digest,Volume:3 21-26July1996 Pages:1802 - 1805 vol.3.

6. "Navy Fact File. United States Navy", [28 June 2001]. Accessed on: [12-062021]. Available: https://nuke.fas.org/guide/usa/c3i/fs_clam_lake_elf2003.pdf.

7. Shepherd S, Jackson CW, Sharkh SM, Aonuma H, Oliveira EE, Newland PL. "Extremely Low-Frequency Electromagnetic Fields Entrain Locust Wingbeats". Bioelectromagnetics. 2021 May;42(4):296-308. doi: 10.1002/bem.22336. Epub 2021 Apr 6. PMID: 33822398

8. Sakhaie MH, Soleimani M, Pourheydar B, Majd Z, Atefimanesh P, Asl SS, Mehdizadeh M. Effects of Extremely Low-Frequency Electromagnetic Fields on Neurogenesis and Cognitive Behavior in an Experimental Model of Hippocampal Injury. Behav Neurol. 2017;2017:9194261. doi: 10.1155/2017/9194261. Epub 2017 Nov 12. PMID: 29259353; PMCID: PMC5702423 
9. Bernstein, S.L, M.L Burrows, J.E Evans, A.S Griffiths, D.A McNeill, C.W Niessen, I Richer, D.P White, and D.K Willim. "Long-Range Communications at Extremely Low Frequencies." Proceedings of the leee 62, no. 3 (1974): 292-312

10. Global geolocation of intense lightning strokes associated with TLEs based on ELF measurements from single station. 2004, 23rd IEEE Convention of Electrical and Electronics Engineers in Israel.

11. Stanford VLF Group. "Introduction to VLF". [Accessed on: 11-30-2021]. Available: https://vlfstanford.ku.edu.tr/research_topic_inlin/introduction-vlf/

12. Howstuffworks. "Resonance", [06-30-2020]. [Accessed: 11-30-2020]. Available: https://science.howstuffworks.com/resonance-info.htm

13. NASA. "Schumann Resonance", [05-28-2013]. [Accessed on: 11-30-2021]. Available: https://www.nasa.gov/mission_pages/sunearth/news/gallery/schumannresonance.html

14.Zeitschrift für Naturforschung A, Volume 7, Issue 2, pp.149-154.

15. Koenig,.H. , Atmospherics geringster frequenzen, Dissertation an der Technischen Hochschule Miinchen. Dec. 1958.

16. Koenig, H.Atmospherics geringster frequenzen, Z. Angew. July. 1959 Phys. II, No.7, 264-274..

17. Koenig, H., E. Haine, and C. H. Antoniadis, Messung von 'atmospherics' geringster frequenzen in Bonn, Z. Angew. Aug. 1967 Phys. 13, No. 8, 364-367.

18. Balser, M., and c. A. Wagner Observations of earthionosphere cavity resonances, Nov. 1960, Nature 188, 638-641.

19. Fournier, II. Some aspects of the first high-frequency geomagnetic recordings obtained at Garchy, Aug. 17, 1960, C. R. Acad. Sci. (France) 251, No. 7, 962964.

20. Benoit, R., and A. Houri, Propagation of very-low-frequencies in the earthionosphere system, 1961, Ann. Geophys. (France) 17, No.4, 370-373.

21. Benoit, R., and A. Houri Power spectrum measurements of geophysical noise. Application to earth-to-ionosphere cavity, Nov. 5, 1962, C. R. Acad. Sci. (France) 255, 2496-2498.

22. Polk, c., and F. Fitchen 'Schumann resonances o.f the ea;th-ionosphere cavityextremely low frequency receptIOn at Kingston, R.I., 1. Res. NBS 66D (Radio Prop.), (May-June 1962), No. 3 313-318.

23. Gendrin, R., and R. Stefant Magnetic records between 0.2 and 30 cis, AGARD Conference on Propagation of Radio Frequencies Below 300 kc/s, Munich, Germany,Sept. 1962a.

24. Balser, M., and C. A. Wagner Diurnal power variations of the earth-ionosphere cavity modes and their relationship to worldwide thunderstorm activity, 1. Geophys. Feb. 1962a, Res. 67, No.2, 619-625.

25. Rycroft, M. J. Low frequency disturbances of natural origin of the electric and magneti c fi elds of the earth, Ph.D. Thesis, University of Ca.IPbridge, Dec. 1963 
26. Chapman, F. W. and D. L. Jones, Observations of earth-ionosphere cavity resonances and thelr interpretation in terms of a two-layer ionosphere model, Nov. 1964b, Radio Sci., 1. Res. NBS 68D, 1177-1185.

27. Williams,E. R. The Schumann resonance: A global tropical thermometer, Science, 1992, 256, 1184.

28. Sátori, G., J. Szendrői, and J. Verő (1996), Monitoring Schumann resonances. I. Methodology, J. Atmos. Terr. Phys., 58, 1475-1481.

29. Price, C., M. Finkelstein, B. Starobinets, and E. Williams (1999), A new Schumann Resonance station in the Negev desert for monitoring global lightning activity, in Proceedings of 11th International Conference on Atmospheric Electricity, Guntersville, Alabama, June 7-11, 1999, pp. 695-697, NASA, Marshall Space Flight Center, Alabama.

30. Hobara, Y., N. Iwasaki, T. Hayashida, T. Tsuchiya, E. R. Williams, M. Sera, Y. Ikegami, and M. Hayakawa, New ELF observation site in Moshiri, Hokkaido, Japan, and the results of preliminary data analysis, J. Atmos.

Electr.,2000, 20(2), 99-109.

31. Andrzej Kulak,Jerzy Kubisz,Slawomir Klucjasz,Adam Michalec,Janusz Mlynarczyk,Zenon Nieckarz,Michal Ostrowski,Stanislaw Zieba. Extremely low frequency electromagnetic field measurements at the Hylaty station and methodology of signal analysis. Published on 17 May 2014

32. Crecraft, David; Gorham, David (2003). Electronics, 2nd Ed. CRC Press. p. 168. ISBN 978-0748770366.

33. https://learning.oreilly.com/library/view/understand-electronics2nd/9780750653190/B9780750653190500197.xhtml. [Accessed on 11/29/2021]

34. Clayton, G. B., et al. Operational Amplifiers, Elsevier Science \& Technology, 2003. ProQuest Ebook Central, https://ebookcentral.proquest.com/lib/wvu/detail.action?doclD=294298.

35. ElectronicTutorials, "Operational Amplifier Basics"”, [05-01-2020]. [Accessed on 11-30-2020]. [Online]. Available: https://www.electronicstutorials.ws/opamp/opamp_1.html

36. Laser \& Electronics. "In-House Rapid PCB Prototyping". [Accessed on: 11-302021] Available: https://www.lpkfusa.com/product_catalog/

37. Circuits Today. "Instrumentation Amplifier", [08-14-2017]. [Accessed on: 11-302020]. [Online]. Available: https://www.circuitstoday.com/instrumentationamplifier

38. Analog Electronics. "AD8421". [Accessed on: 11-30-2020]. [Online]. Available: https://www.analog.com/media/en/technical-documentation/datasheets/AD8421.pdf

39. Don Lancaster, Active-Filter Cookbook, Howard W. Sams and Co., 1975 ISBN 0672-21168-8 pages 8-10. 
40. Learning about Electronics. [Accessed on: 11-30-2021]. [Online]. Available: http://www.learningaboutelectronics.com/Articles/What-is-an-ideal-op-amp.php

41. Dummies. "Electronics Components: Closed Loop Amplifiers", [03-26-2016]. [Accessed on: 11-30-2020]. [Online]. Available: https://www.dummies.com/programming/electronics/components/electronicscomponents-closed-loop-amplifiers

42. Wikiwand. "Operational amplifier". [Accessed on: 11-30-2021]. [Online]. Available: https://www.wikiwand.com/en/Operational_amplifier

43. "Analog Devices AN1264". [Accessed on: 11-30-2021]. [Online]. Available: https://www.analog.com/media/en/technical-documentation/application-notes/AN1264.pdf

44. Analog Devices. "Passive Low Pass Filter", [05-01-2020]. [Accessed on: 11-302021]. [Online]. Available: https://www.electronics-tutorials.ws/filter/filter_2.html

45. ECStudio. "RC Passive Low-Pass Filter". [Accessed on: 11-30-2021]. [Online]. Available: https://ecstudiosystems.com/discover/textbooks/basicelectronics/filters/passive-low-pass-filter/

46. Electronics Hub. "Active Low Pass Filter", [10-06-2021]. [Accessed on: 11-302021]. Available: https://www.electronicshub.org/active-low-pass-filter/

47. Electronics Tutorials. "Active Low Pass Filters", [11-11-2020]. [Accessed on: 1130-2021]. Available: https://www.electronics-tutorials.ws/filter/filter_5.html

48. "Analog Devices AD8510", [10-22-2007]. [Accessed on: 11-30-2021]. Available: https://www.analog.com/en/products/ad8510.html\#product-overview

49. GroupDIY. "Mixer internal phantom power blocking and adding HPF", [12-302020]. [Accessed on: 11-30-2020]. Available: https://groupdiy.com/threads/mixerinternal-phantom-power-blocking-and-adding-hpf.76672/

50. Maxin integrated. "GLOSSARY DEFINITION FOR NON-INVERTING OP AMP". [Accessed on: 11-30-2021]. Available:

https://www.maximintegrated.com/en/glossary/definitions.mvp/term/NonInverting\%200p\%20Amp/gpk/1219

51. CircuitBread. "What is Common-Mode Rejection Ratio in Op-amps?", [01-112021]. [Accessed on: 11-30-2021]. [Online]. Available:

https://www.circuitbread.com/ee-faq/what-is-common-mode-rejection-ratio-in-opamps

52. Wikipedia, "Sallen-Key topology", [05-31-2021]. [Accessed on: 11-30-2021]. [Online]. Available: https://en.wikipedia.org/wiki/Sallen\%E2\%80\%93Key_topology

53. Laser \& Electronics. "LPKF ProtoMats PCB Milling Systems". [Accessed on: 1130-2021]. [Online]. Available: https://www.lpkf.com/en/industriestechnologies/research-in-house-pcb-prototyping/products/lpkf-protomat TSM, "ProtoLaser ST. [Accessed on: 11-30-2021]. Available: https://tsmgroup.vn/danh-muc/protolaser-st/ 


\section{Chapter-6}

\section{Appendix -1:}

An Inductor-Capacitor(LC) circuit is connected before amplifier which acts as an antenna to detect $7.8 \mathrm{~Hz}$ signals. This circuit is obtained from Dr. Roy Nutter. The values of Inductance and Capacitance used here are $1.59 \mathrm{H}$, and $268 \mathrm{uF}$ respectively. Resonant frequency is calculated using the formula $\frac{1}{2 * \pi * \sqrt{L * C}}$

Calculating the resonant frequency using the inductor and Capacitor values,

Resonant Frequency $=\frac{1}{2 * \pi * \sqrt{1.59 * 268 *\left(10^{-6}\right)}}=7.8 \mathrm{~Hz}$.

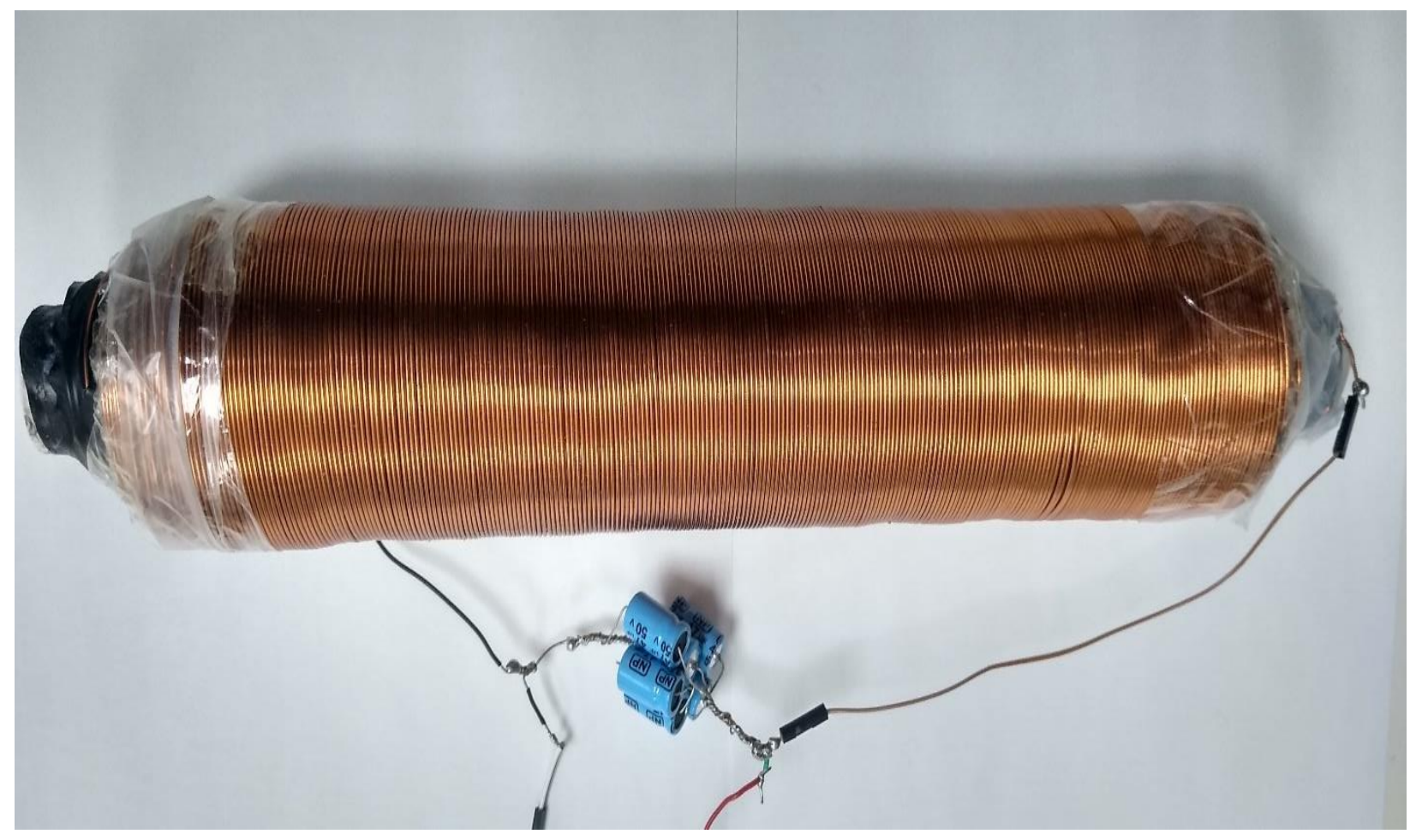

Figure 33, Inductor - Capacitor(LC) circuit

\section{Appendix -2:}

WVU iLabs has Innovation Hub which is a suite of design, machining, building and fabrication techniques for creating prototypes. Innovation Hub is located in Engineering sciences building of Evansdale campus. The Lane Innovation Hub is open to individuals and groups from both inside the WVU community and broader state community. Users of the Lane Innovation Hub Maker Space can get equipment training to develop their ideas into working prototypes or use the Lane Innovation Hub Service Center to have components made for research or entrepreneurial projects. Innovation Hub has Rapid 
Prototyping Lab (ESB G85), Advanced Manufacturing Lab (ESB G86), Electronics Prototyping Lab (ESB G75E) Metal 3D Printing Lab (ESB G59).

PCB shown in Figure 21, is manufactured using ProtoMat and ProtoLaser ST. Statler college students get free training on the equipment. Students can reserve the equipment and can manufacture their own PCB. There are series of steps to follow to manufacture the PCB using those machines. Before going to ProtoMat design the board using a PC Board designing software. In this work EAGLE software provided by AUTODESK (software corporation that makes software products and services) is used. Gerber files are generated from Eagle, which contain information about through holes, vias, copper layer, soldering masks and traces. These files are utilized by ProtoMat and ProtoLaser machines in the process. CircuitProPM, CircuitProPL software's are used to process the data from Gerber files and send data to ProtoMat and ProtoLaser.

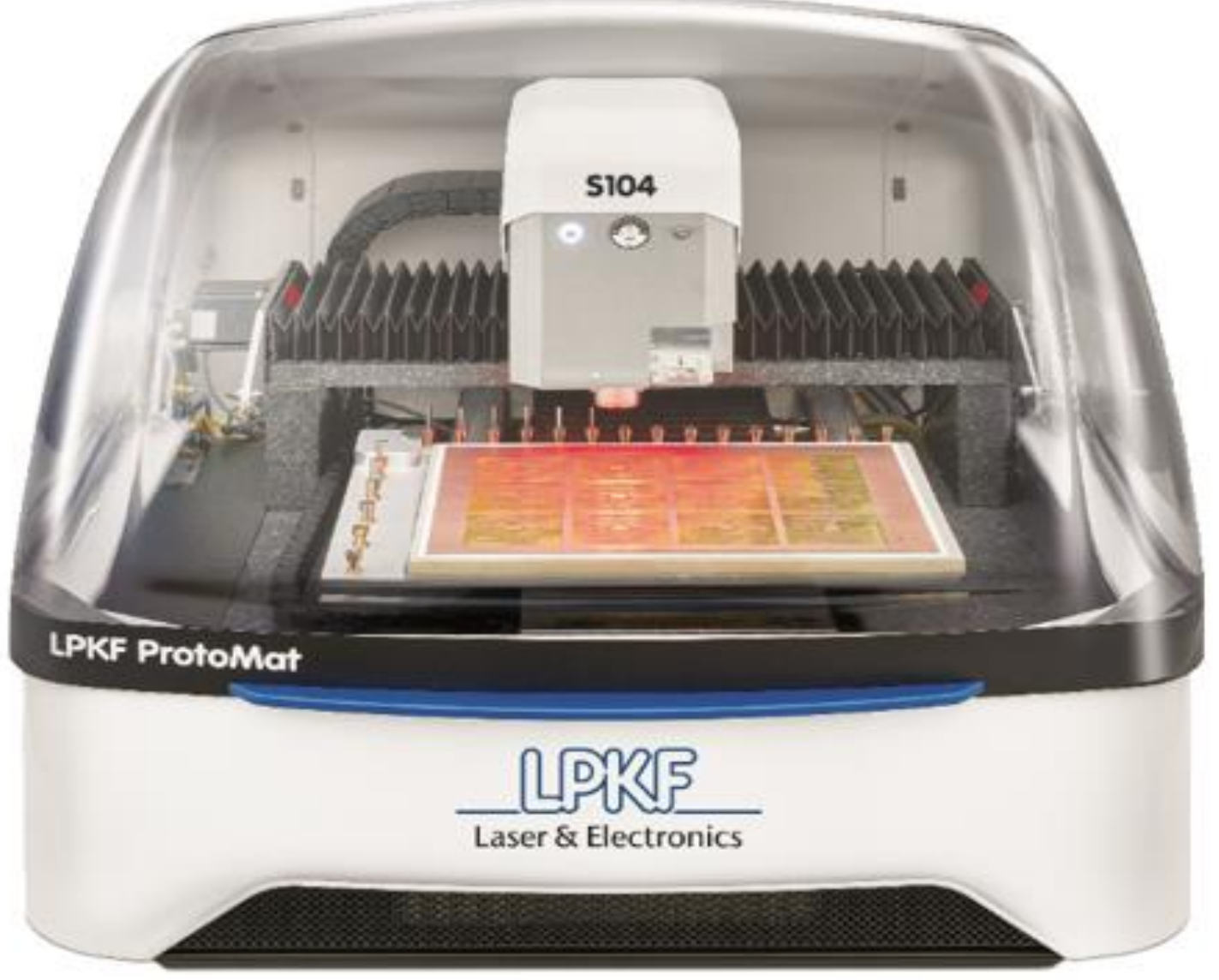

Figure 34, ProtoMat[53] 


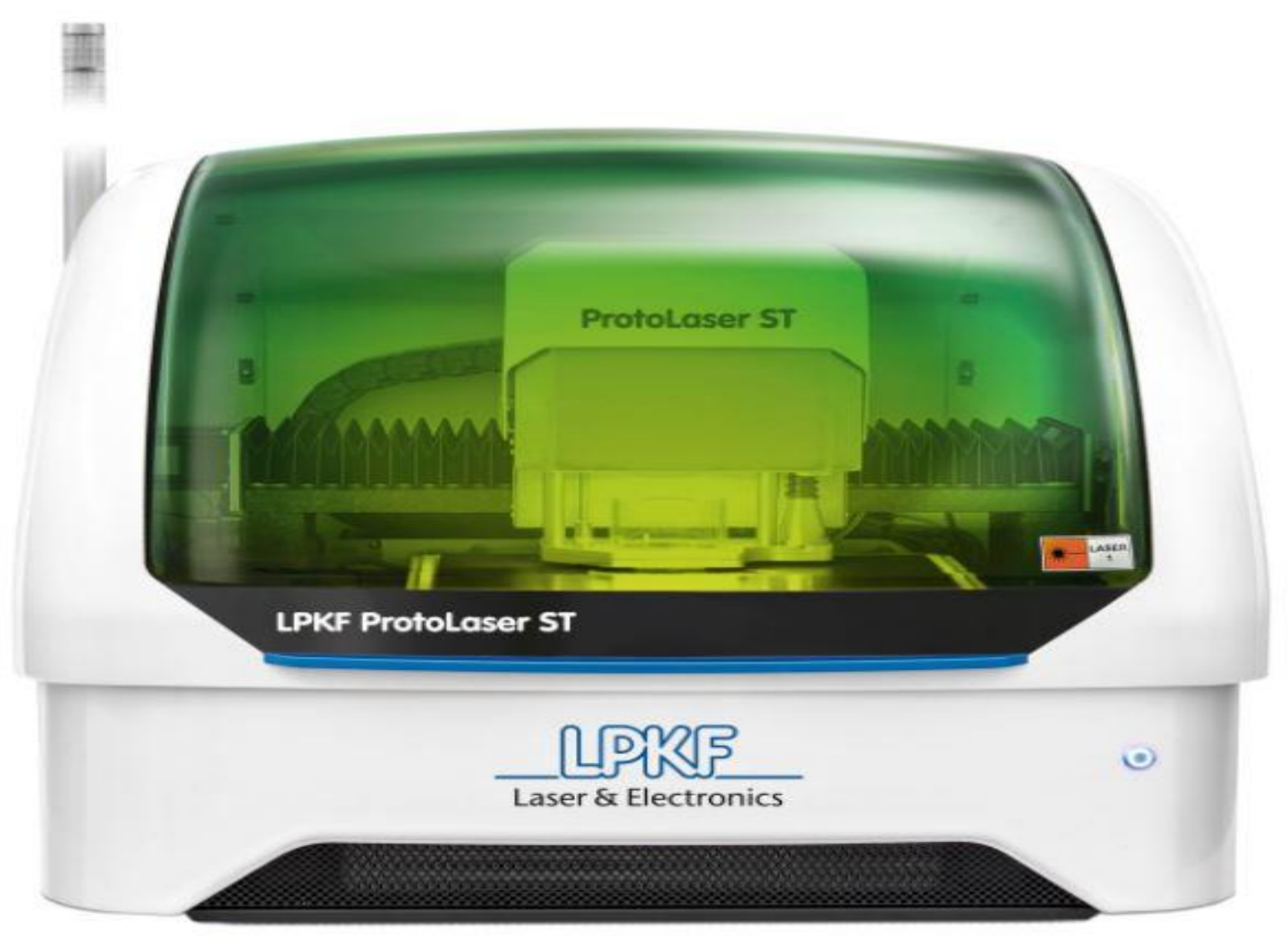

Figure 35, ProtoLaser[54]

Following steps are performed to produce a double-sided PCB with galvanic through-hole plating:

- Switching on the ProtoMat

- Importing the data in CircuitPro PM

- Assigning holes to the layer DrillUnplated in CircuitPro PM

- Multiplying the layout in CircuitPro PM

- Creating fiducials in CircuitPro PM

- Saving the file in CircuitPro PM

- Generating toolpaths in CircuitPro PM

- Processing a PCB(with ProtoMat)

- Galvanic through-hole plating the PCB

- Preparing the data in CircuitPro PL

- Structuring the PCB(with ProtoLaser)

- Drilling unplated through holes and cutting out the PCB(with ProtoMat)

- Cleaning the PCB

- Cleaning the ProtoMat. [36] 